\title{
Aging-associated inflammation promotes selection for adaptive oncogenic events in B cell progenitors
}

\author{
Curtis J. Henry, ${ }^{1,2}$ Matias Casás-Selves, ${ }^{1}$ Jihye Kim, ${ }^{3}$ Vadym Zaberezhnyy, ${ }^{1}$ Leila Aghili, ${ }^{1}$ Ashley E. Daniel, ${ }^{1}$ Linda Jimenez, ${ }^{1}$ \\ Tania Azam, ${ }^{4}$ Eoin N. McNamee, ${ }^{5}$ Eric T. Clambey, ${ }^{5}$ Jelena Klawitter, ${ }^{5}$ Natalie J. Serkova, ${ }^{5}$ Aik Choon Tan, ${ }^{3}$ \\ Charles A. Dinarello, ${ }^{4,6}$ and James DeGregori ${ }^{1,2}$ \\ 'Department of Biochemistry and Molecular Genetics, University of Colorado Anschutz Medical Campus (AMC), Aurora, Colorado, USA. ${ }^{2}$ Integrated Department of Immunology, National Jewish Health, \\ Denver, Colorado, USA. ${ }^{3}$ Division of Medical Oncology, ${ }^{4}$ Division of Infectious Disease, and ${ }^{5}$ Department of Anesthesiology, University of Colorado AMC, Aurora, Colorado, USA. ${ }^{6}$ Department of Medicine, \\ Radboud University Medical Center, Nijmegen, Netherlands.
}

\begin{abstract}
The incidence of cancer is higher in the elderly; however, many of the underlying mechanisms for this association remain unexplored. Here, we have shown that B cell progenitors in old mice exhibit marked signaling, gene expression, and metabolic defects. Moreover, B cell progenitors that developed from hematopoietic stem cells (HSCs) transferred from young mice into aged animals exhibited similar fitness defects. We further demonstrated that ectopic expression of the oncogenes $B C R$ - $A B L$, $N R A S^{V 12}$, or Myc restored B cell progenitor fitness, leading to selection for oncogenically initiated cells and leukemogenesis specifically in the context of an aged hematopoietic system. Aging was associated with increased inflammation in the BM microenvironment, and induction of inflammation in young mice phenocopied aging-associated B lymphopoiesis. Conversely, a reduction of inflammation in aged mice via transgenic expression of $\alpha-1$-antitrypsin or IL-37 preserved the function of B cell progenitors and prevented $N_{R A S}{ }^{12}$-mediated oncogenesis. We conclude that chronic inflammatory microenvironments in old age lead to reductions in the fitness of B cell progenitor populations. This reduced progenitor pool fitness engenders selection for cells harboring oncogenic mutations, in part due to their ability to correct aging-associated functional defects. Thus, modulation of inflammation - a common feature of aging - has the potential to limit aging-associated oncogenesis.
\end{abstract}

\section{Introduction}

The incidence of many human diseases, including cancers, increases with age (1). The aging process is associated with functional impairment at the tissue, cellular, and molecular levels (2). Aging-associated changes are particularly evident in the BM of mice and humans, manifesting as reductions in BM density, changes in vascularization, and alterations in the composition of accessory and structural cells (3).

The function of hematopoietic stem and progenitor cells (HSPCs) significantly declines with age in both mice and humans (4-7). In competitive transplantation assays, old hematopoietic stem cells (HSCs) exhibit reduced reconstitution potential on a per-cell basis (a measure of "cellular fitness") when compared with HSCs isolated from young mice. Aged HSCs also exhibit altered metabolism and localization in the $\mathrm{BM}$ as well as reduced BM homing potential upon transplantation (8-10). Aging-associated hematopoietic defects are particularly apparent in the lymphoid lineages, which in mice and humans can be partly attributed to the skewing of the HSC repertoire toward myeloid-biased HSCs $(6,10-13)$. Additional studies have indicated that the aged BM microenvironment can contribute to reductions in B lymphopoiesis $(14,15)$. Notably, we have previously shown that declining $B$ lymphoid progenitor fitness in old mice promotes selection for the

Conflict of interest: The authors have declared that no conflict of interest exists. Submitted: July 15, 2015; Accepted: October 1, 2015

Reference information: J Clin Invest. 2015;125(12):4666-4680. doi:10.1172/JCI83024.
$B C R-A B L$ oncogene due to its ability to correct aging-associated defects in cell signaling (16).

More recently, it has been demonstrated that inflammatory cytokines regulate the function of hematopoietic progenitor cells. TNF- $\alpha$ and TGF- $\beta$ (at high concentrations) have been shown to suppress HSC activity $(17,18)$, whereas IFN- $\alpha$, IFN- $\gamma$, and TGF- $\beta$ (at low concentrations) activate HSC proliferation (19-21). Moreover, inflammation has been shown to directly impair B lymphopoiesis (and thus favor myelopoiesis) by preventing B progenitor localization to the IL-7-rich niches required for B cell development (22-24). In aged mice, the production of TNF- $\alpha$ by agingassociated B cells impairs B lymphopoiesis $(14,25)$, and the myeloid-biasing of the hematopoietic compartment with age is in part regulated through the actions of TGF- $\beta 1$ (18). Whereas inflammation is important for survival in youth to combat infections and repair tissues, it can have adverse effects on aged individuals (26, 27). Indeed, older humans typically present a subclinical systemic chronic inflammatory status termed "inflamm-aging," which has been postulated to contribute to the development of a variety of aging-associated diseases such as Alzheimer's disease, cardiovascular disease, and cancer (28-31).

Current paradigms attribute the association between aging and cancer primarily to the progressive accrual of oncogenic mutations that are widely thought to be the rate-limiting events in the generation of most cancers (32-34). Predominant models of carcinogenesis mostly assume that mutations convey defined fitness effects on transformed cells; however, this idea contra- 
A Purine

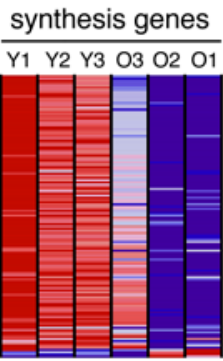

Pyrimidine synthesis genes

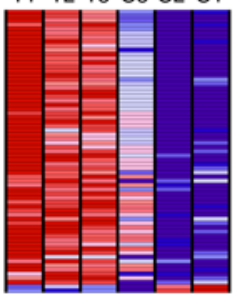

B

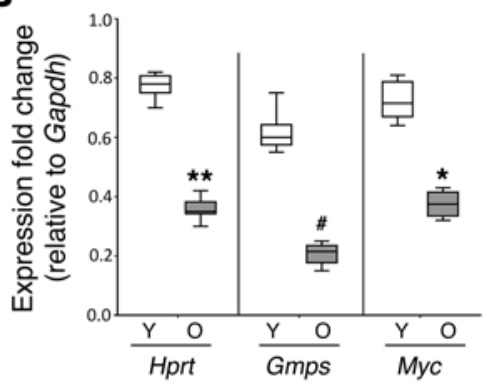

C

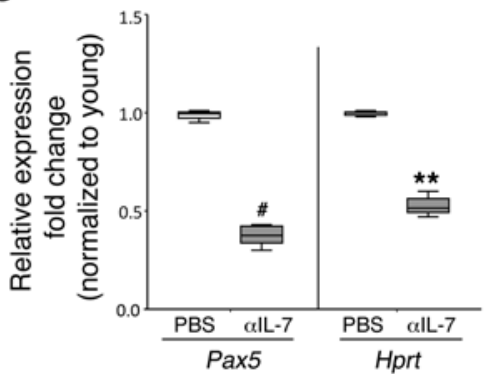

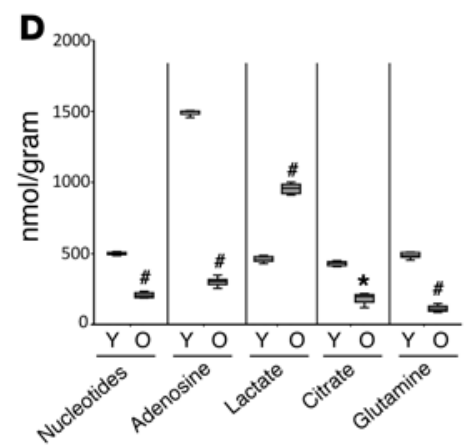
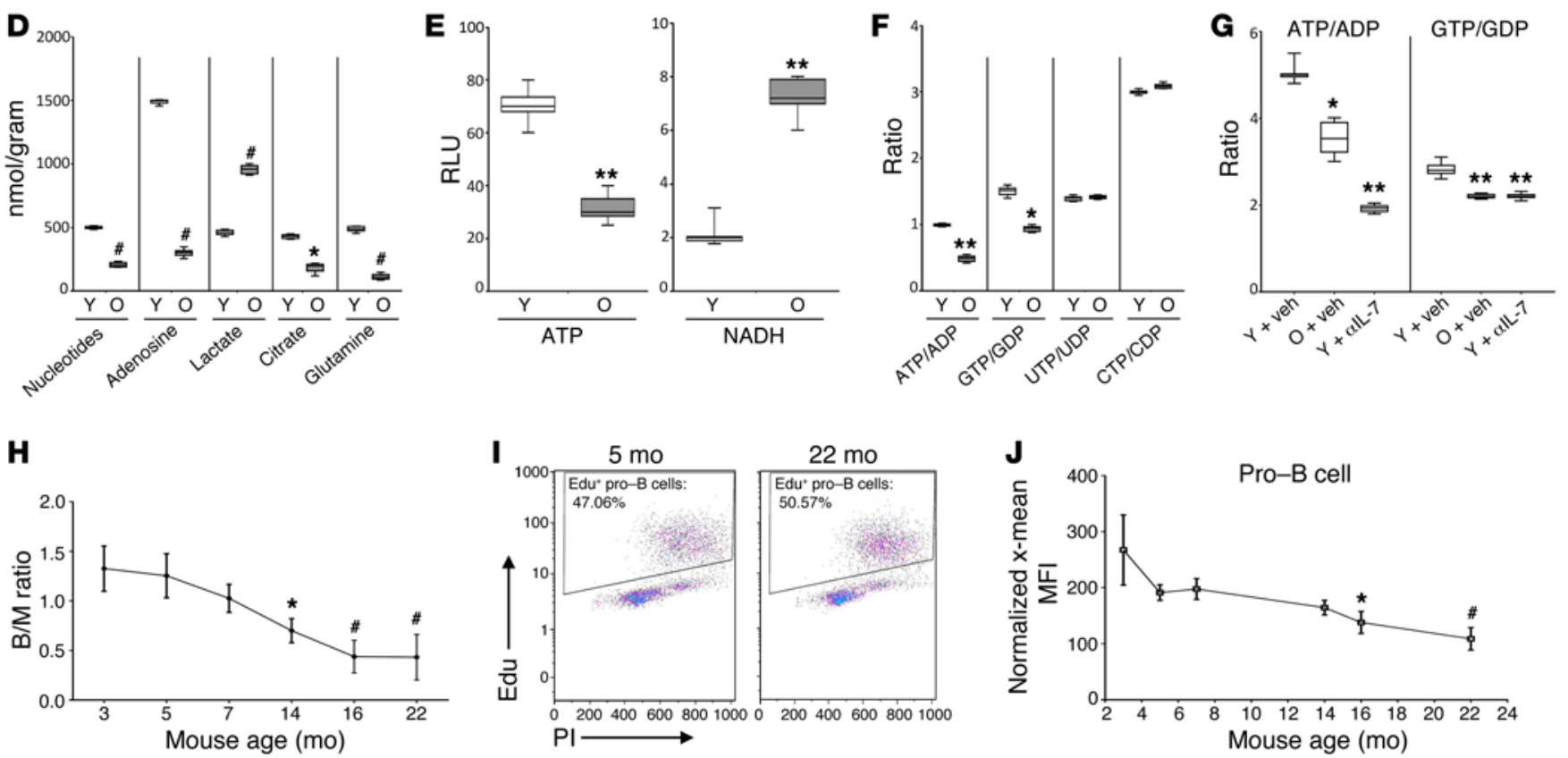

Figure 1. Impaired metabolism, nucleotide anabolism, and cell cycling accompany aging B lymphopoiesis. (A) Nucleotide synthesis GSEA sets were derived from microarray analysis of B progenitors isolated from young and old BALB/c mice. Y1, young mouse \#1; 01, old mouse \#1. (B) Hprt, Gmps, and Myc expression in sorted young and old pro-B cells was determined using qPCR. Values represent the mean \pm SEM of 4 independent experiments (8 donor mice/age group). (C) Young BALB/c mice were injected with 1x PBS or IL-7-neutralizing Abs (alL-7) every 4 days for 2 weeks, and Pax5 and Hprt expression was determined by qPCR in B220-purified B progenitors. Values represent the mean \pm SEM of 3 independent experiments (6 mice/age group). (D) B progenitors were isolated from young and old BALB/c mice, and NMR metabolomics was performed. Values represent the mean \pm SEM of 2 independent experiments (8 donor mice/age group). (E) ATP and NADH levels were determined in B progenitors isolated from young and old BALB/c mice. Values represent the mean \pm SEM of 3 independent experiments ( 9 donor mice/age group). (F) Samples used in $\mathbf{D}$ were analyzed by mass spectrometry for relative nucleotide levels.(C) Young and old BALB/c mice were treated with 1× PBS (Veh.), or young mice were treated with IL-7-neutralizing Abs as described in C, and the energy balance of purine nucleotides in $\mathrm{B}_{22 \mathrm{O}^{+}}$cells was determined by mass spectrometry. Values represent the mean $\pm \mathrm{SEM}$ of 3 independent experiments (4 donor mice/group). (H-J) C57BL/6 mice were injected with EdU, BM was harvested 2 hours later, and cell-cycle analysis in pro-B cells was performed. (H) B220 $/ \mathrm{MAC1}^{+}$(B/M) cell ratio. (I) Representative cell-cycle profiles. (J) Normalized x-mean MFI of EdU+ cell populations for pro-B cells. Statistical analyses in $\mathbf{H}$ and $\mathbf{J}$ are relative to the levels observed in $\mathbf{5}$-month-old mice and represent the mean \pm SEM of 3 independent experiments (4 donor mice/age group). ${ }^{*} P<0.05$, ${ }^{* *} P<0.01$, and ${ }^{\#} P<0.001$, by Student's $t$ test relative to young controls for each experiment. $Y$, young; 0 , old.

dicts evolutionary theory, which holds that fitness is dictated by the interaction of a genotype-defined phenotype with the environment (35). Similarly, the somatic mutation theory of aging largely attributes age-dependent tissue decline to the accumulation of somatic mutations throughout life $(2,32,33,36)$. Our laboratory has computationally modeled fitness changes and somatic evolution in HSC pools during life to demonstrate that mutation accumulation alone cannot account for either HSC fitness decline or late-life clonal evolution (35). Importantly, these modeling studies demonstrate that age-dependent alterations in the tissue microenvironment are necessary for both HSC fitness decline and clonal evolution (where cellular "fitness" is defined as a measure of the ability of stem/progenitor cells of a certain epigenotype/genotype to transmit this type to subsequent cell generations).

Given the strong correlations between advanced age, chronic systemic inflammation, and cancer incidence in mammals, in this study we sought to determine how aging-associated inflammation impacts lymphoid progenitor populations and how this state influences the evolution of leukemias. Using transgenic expression of two different proteins, $\alpha$-1-antitrypsin (AAT) and IL-37, in order to reduce inflammation in old mice, we show that preventing aging-associated reductions in B progenitor fitness abrogates selection for oncogene-initiated progenitors. 


\section{Results}

Metabolic and cell-cycle defects accompany aging B lymphopoiesis. In order to understand the mechanism underlying declining $\mathrm{B}$ lymphopoiesis in old age, we performed microarray analysis on combined pro- and pre-B cell populations isolated from young (2-month-old) and old (24-month-old) mice. Gene set enrichment analysis (GSEA) revealed that aging $\mathrm{B}$ lymphopoiesis is accompanied by significant reductions in purine and pyrimidine metabolism (Figure 1A and Supplemental Figure 1A; supplemental material available online with this article; doi:10.1172/ JCI83024DS1). The aging-associated decreases in the expression of the key purine synthesis genes hypoxanthine-guanine phosphoribosyltransferase (Hprt) and guanine monophosphate synthase (Gmps) were confirmed using quantitative PCR (qPCR) analysis in sorted pro-B cell populations (Figure 1B).

IL-7 signaling decreases with age in B cell progenitors $(16,37)$. Similar to previous reports (38), inhibiting IL-7R signaling by injecting mice with IL-7 neutralizing Abs ( $\alpha$ IL-7) significantly decreased the expression of Pax 5 in pro-B cells (Figure 1C). Notably, reducing IL-7R signaling also significantly decreased Hprt gene expression in pro-B cells (Figure 1C), suggesting that aging-associated reductions in purine synthesis gene expression can be explained, at least in part, by an impairment of IL-7R signaling.

Our microarray analysis also indicated that mitochondrial dysfunction accompanied aging B lymphopoiesis $\left(P=7.01 \times 10^{-7}\right.$ for mitochondrial dysfunction using Ingenuity Pathway Analysis [IPA]), suggesting that metabolic changes also accompany aging B lymphopoiesis. Indeed, nuclear magnetic resonance (NMR) analysis revealed significant decreases in total nucleotide and adenosine levels in aged B cell progenitors (Figure 1D), consistent with reduced purine synthesis resulting from decreased expression of genes such as Hprt and Gmps. Furthermore, other metabolic intermediates such as citrate (a key TCA cycle intermediate) and glutamine (an amino acid that can enter the TCA cycle) levels were significantly decreased with age in B cell progenitors (Figure 1D). Importantly, lactic acid (a marker for increased glycolytic output and mitochondrial impairment) was also found to be increased in old B cell progenitors (Figure 1D). These metabolic perturbations coincided with significant reductions in ATP, reduced ATP/ADP and GTP/GDP ratios, elevated NADH levels, and significant reductions in mitochondrial ROS levels in aged $\mathrm{B}$ cell progenitors (Figure 1, E and F, and Supplemental Figure 1B). Reduced ATP/ADP and GTP/GDP ratios (reduced energy balances) could be recapitulated in B cell progenitors in young mice by inhibiting IL-7R signaling (Figure $1 G$ ).

Analysis of total STAT5a/b protein levels in B cell progenitors revealed that aged pro- and pre-B cell progenitors had significantly lower STAT5a/b protein levels relative to their young counterparts (Supplemental Figure 2A), correlating with an overall reduction in phosphorylated STAT5a (p-STAT5a) protein levels (Supplemental Figure 2B). Notably, impairments in B cell production, STAT5-mediated signaling, and the expression of key genes involved in purine synthesis (Gmps), B cell development (Ebf), and proliferation $(M y c)$ were not reversed by transplantation into irradiated young mice (Supplemental Figure 2, C-E). These observations indicate that aging-associated reductions in IL-7R signaling in $\mathrm{B}$ cell progenitors result, at least in part, from the reduced avail- ability of key signaling intermediates, and a young nonhematopoietic microenvironment cannot correct aging-associated defects in B cell progenitors that develop from the transplanted old HSC.

Given the extensive metabolic changes associated with aging B lymphopoiesis as well as the findings of previous studies demonstrating increased replicative stress and cell-cycle defects in HSCs from old mice (39), we determined the consequences of these perturbations on cell-cycle progression. Mice of various ages were injected with the nucleotide analog 5-ethynyl-2'-deoxyuridine (EdU). We observed significant reductions in B lymphopoiesis in mice between 14 and 22 months of age, as indicated by a decrease in the ratio of $\mathrm{B}$ cell $\left(\mathrm{B} 22 \mathrm{O}^{+}\right)$to myeloid cell $\left(\mathrm{MAC1}^{+}\right)$progenitors (Figure 1H). Notably, aged pro-B cell progenitors exhibited a significant reduction in the rate of S-phase progression (Figure 1, I and J); however, we did not observe increased apoptosis in aged B progenitor populations (Supplemental Figure 2G). Overall, these results reveal that aging $\mathrm{B}$ lymphopoiesis is accompanied by extensive metabolic changes that culminate in defective cell-cycle progression in B cell progenitors. Since these alterations coincide with the reduced competitive potential of old B progenitors (40) and impairing IL-7R signaling is sufficient to reduce the function of B cell progenitors and impair B lymphopoiesis, we conclude that these signaling, metabolic, and cell-cycle defects are contributing to an age-associated decline in B progenitor fitness.

Oncogenic mutations correct aging-associated functional defects in $B$ progenitors. Given that $B C R-A B L$ expression corrects defective IL-7R-mediated signaling in aged $B$ cell progenitors, leading to increased leukemogenesis in aged backgrounds (16), we determined whether $B C R-A B L$ expression reverses aging-associated defects in key genes involved in metabolism and DNA replication. Since $B C R$ $A B L$ expression activates RAS and MYC (41), we also asked whether the NRAS ${ }^{V 12}$ and MYC oncogenes are adaptive in aged backgrounds by correcting aging-associated $\mathrm{B}$ progenitor fitness defects.

Using the $\mathrm{Ba} / \mathrm{F} 3$ pro- $\mathrm{B}$ cell line, we found that directly inhibiting cytokine receptor signaling on $\mathrm{B}$ lineage cells in vitro was sufficient to reduce the expression of key nucleotide synthesis genes, as reducing IL-3-mediated stimulation of the $\mathrm{Ba} / \mathrm{F} 3$ pro-B cell line impaired STAT5 phosphorylation (Figure $2 \mathrm{~A}$ ) and expression of $M y c$ (Figure 2B) and Hprt (Figure 2B). Importantly, the expression of oncogenic BCR-ABL, NRAS V12, and Myc maintained STAT5 phosphorylation and $M y c$ and Hprt expression levels in $\mathrm{Ba} / \mathrm{F} 3$ cells despite cytokine withdrawal (Figure 2, A and B), indicating a direct ability of these oncogenes to rescue cytokine receptor signaling defects.

In order to study oncogenic adaptation in vivo, hematopoietic progenitor cells from young and old mice were retrovirally transduced to introduce the oncogenes $B C R-A B L, N R A S^{V 12}$, or $M y c$ and transplanted into sublethally irradiated young recipient mice. Three weeks after transplantation, oncogene or vector bearing pro-B cell progenitors $\left(\mathrm{GFP}^{+}\right)$were flow sorted from recipient mice and their signaling and gene expression profiles determined. The expression of oncogenic BCR-ABL, NRAS $S^{V 12}$, and $M y c$ each led to restoration of p-STAT5 activation in old pro-B progenitors (Figure $2 \mathrm{C}$ ). In contrast, expression of these oncogenes did not lead to significant alterations in p-STAT5 levels in young pro-B cells, for which signaling was already high. We also observed that oncogenic $B C R-A B L, N R A S^{V 12}$, and $M y c$ restored youthful expression 
A

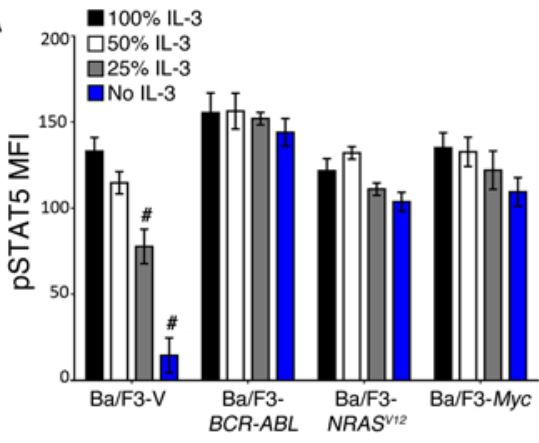

B

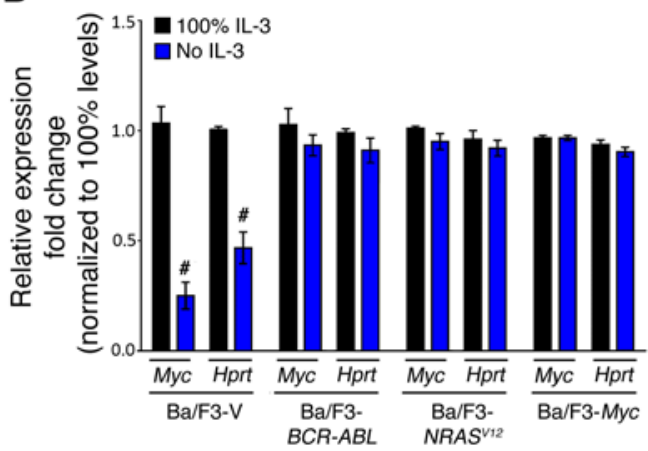

C

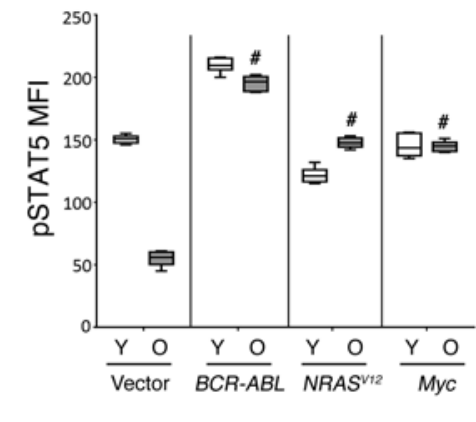

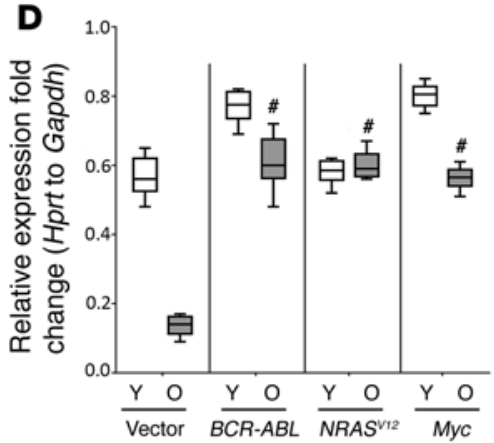

G

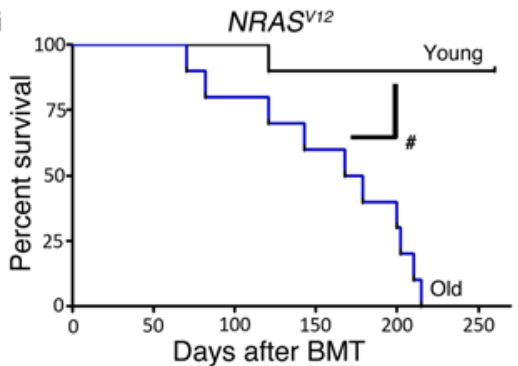

E
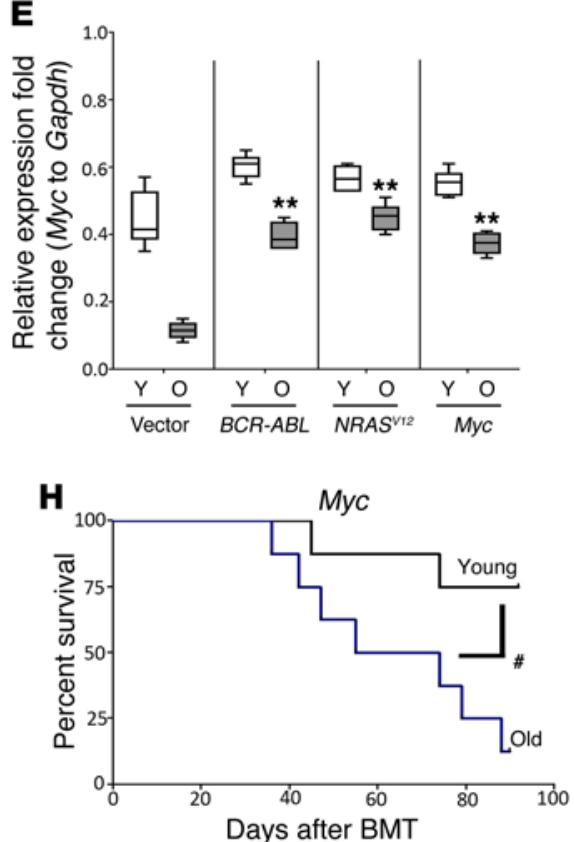

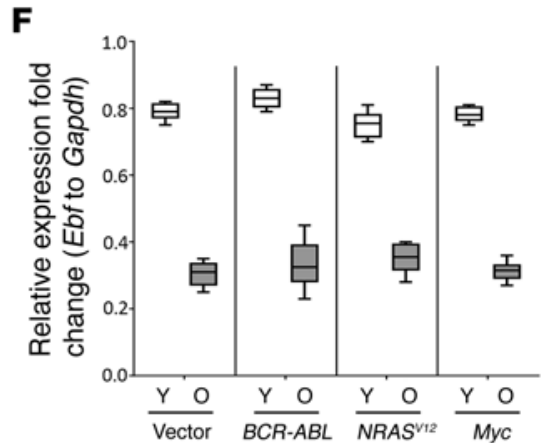

I

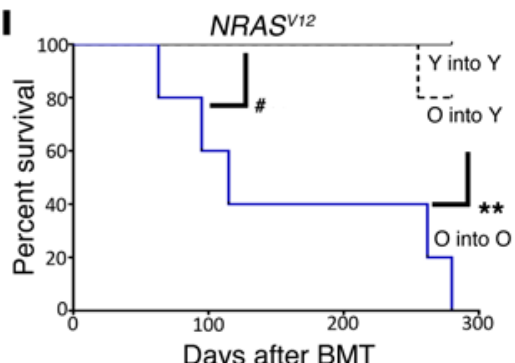

Figure 2. Oncogenic mutations correct aging-associated functional defects in B progenitors, leading to increased leukemogenesis. (A) Ba/ $F 3$ cells expressing vector $(V)$ or oncogenes ( $\left.B C R-A B L, N R A S^{V 12}, M y c\right)$ were grown in various concentrations of IL-3 for 24 hours, and STAT5 activation in these cells was determined by flow cytometry. (B) Ba/F3 cells were grown overnight in media containing or lacking IL-3, and expression levels of Myc and $H p r t$ in these cells were determined by qPCR. Values in $\mathbf{A}$ and $\mathbf{B}$ represent the mean \pm SEM of 3 independent experiments ( 9 total samples). (C-F) c-KIT+ BM cells were isolated from young (2-month-old) or old (24-month-old) mice, retrovirally transduced to express vector or oncogenic BCR-ABL, NRAS V12, or Myc (each with coexpressed GFP), and transplanted into sublethally irradiated young BALB/c mice. Three weeks after transplantation, mice were sacrificed, and STAT5 activity (C) and mRNA levels of Hprt, Myc, and Ebf (D-F) were determined in vector-expressing or oncogene-expressing pro-B cell progenitors. Values in C-F represent the mean \pm SEM for more than 5 mice per group. $\left(\mathbf{G}\right.$ and $\mathbf{H}$ ) Young mice were lethally irradiated and transplanted with either $2 \times 10^{6}$ young or old whole BM cells. Four days later, mice reconstituted with young or old BM cells were transplanted, respectively, with young or old c-KIT+ cells expressing oncogenic NRAS ${ }^{112}$ or Myc. Leukemia-free survival is plotted using Kaplan-Meier graphs. Most mice developed B220+CD43+ pro-B cell-like acute lymphoblastic leukemia (ALL) (87\% and $80 \%$ for NRAS- and Myc-driven leukemias, respectively, on the old backgrounds). (I) Young or old mice were treated with busulfan and transplanted with young or old c-KIT+ cells expressing oncogenic NRAS ${ }^{\text {12 }}$. Leukemia-free survival is plotted using Kaplan-Meier graphs. Values in $\mathbf{G}$ and $\mathbf{H}$ represent the mean \pm SEM of 2 independent experiments, with more than 15 mice per group in total, and values in $\mathbf{I}$ represent 5 mice per treatment group. $(\mathbf{A}-\mathbf{F}){ }^{* *} P<0.01$ and ${ }^{\#} P<0.001$, by Student's $t$ test . In $\mathbf{C}-\mathbf{F}$, oncogene-bearing samples were compared with vector-expressing controls (old to old). (G-I) ${ }^{* *} P<0.01$ and ${ }^{\#} P<0.001$, by Cox proportional hazards test. BMT, BM transplantation.

levels of $H p r t$ (Figure 2D) and endogenous $M y c$ (Figure 2E). Interestingly, the defective expression of the B lineage specification genes $E b f$ (Figure 2F) and Pax5 (Supplemental Figure 2F) in aged B cell progenitors was not restored by these oncogenes. From these results, we conclude that $B C R-A B L, N R A S^{V 12}$, and $M y c$ expression restores the key parameters of fitness, but not the differentiation potential, of aged B progenitors.

We next determined whether NRAS $S^{V 12}$ and $M y c$-mediated restoration of impaired fitness in aged B progenitors, by provid- ing a competitive advantage within poorly fit old B progenitor pools, promotes increased leukemogenesis. Lethally irradiated young mice were prepopulated with young or old BM cells to create young and aged hematopoietic systems, respectively, and then transplanted with either young or old BM progenitor cells transduced with the NRAS ${ }^{V 12}$ and $M y c$ oncogenes. Oncogene-driven restoration of B progenitor function in old, but not young, pro-B cells led to increased NRAS ${ }^{V 12}$ - and Myc-mediated leukemogenesis in aged backgrounds. In contrast, leukemogenesis was largely 
suppressed in young hematopoietic contexts (Figure 2, G and $\mathrm{H}$ ). Thus, provision of the oncogenic drivers is insufficient to induce leukemias, as the leukemic potential of the oncogenic mutations is only fully realized in the aged hematopoietic context. Notably, we have previously shown that the differential ability of $B C R-A B L$ to induce leukemias in young and old hematopoietic backgrounds does not require an intact adaptive immune system (16).

We then asked whether the age of the host would similarly impact leukemogenesis; specifically, would transplantation of $N R A S^{V 12}$-transduced old BM into a young recipient still efficiently induce leukemias? In order to reduce the transplant-induced affects on the host BM microenvironment, we used busulfan rather than $\gamma$ irradiation to condition recipient mice prior to transplantation with $N R A S^{V 12}$-transduced BM. Busulfan conditioning induced a mild inflammatory response, as indicated by transient increases in BM and serum IL- 6 and TNF- $\alpha$ levels, which returned to baseline by day 5 after treatment (Supplemental Figure 3, A-D). BM hematopoietic cell populations returned to homeostatic numbers by day 5 after busulfan treatment (Supplemental Figure 3, E-G). In contrast, sublethal irradiation induced greater and more prolonged increases in these cytokines, and B lymphopoiesis was still highly suppressed on day 5 after irradiation (Supplemental Figure 3, A-G). Importantly, transplantation of oncogenic $N R A S^{V 12}$-transduced old $\mathrm{BM}$ progenitors into young mice resulted in significantly less leukemia than their transplantation into old recipient mice (Figure 2I). We tracked the frequencies of $N R A S^{V 12}$-expressing $\left(\mathrm{GFP}^{+}\right) \mathrm{B}$ cells and myeloid cells in peripheral blood over the course of the experiment and found that the percentage of B lineage cells expressing NRAS $S^{V 12}$ was only maintained and amplified in old recipient mice, but not in young recipient mice, even when donor progenitor cells were old (Supplemental Figure 2, H-K). Thus, the age of the microenvironment may be more important than the age of the cells receiving the oncogenic hit in leukemia development. In total, these results reveal that oncogenic BCR-ABL, NRAS V12, and $M y c$ expression correct functional defects in aged pro-B cells, leading to increased selection in aged hematopoietic backgrounds, with subsequent progression to B-lineage leukemias.

Increased inflammation in the BM with age coincides with decreased expression of genes regulating cell-cycle progression in B progenitors. Since inflammation increases with age in most mammals (31) and regulates the function of HSPCs (42), we explored the relationship between aging-associated increases in inflammation and B lymphopoiesis. Principal component analysis (PCA) of the microarray data indicated that gene expression profiles for B progenitors isolated from individual young mice exhibited low intragroup variability; in contrast, B progenitors isolated from old mice exhibited divergent gene expression profiles (Figure 3A). Notably, reductions in E2F and MYC target genes, including genes involved in nucleotide synthesis and cell-cycle progression, coincided with increases in the inflammatory signature (Figure 3B). In order to determine the kinetics of altered inflammation with age, we performed ELISAs for inflammatory cytokines on serum and BM aspirates taken from young, middle-aged, and old mice. Whereas analyses of serum samples revealed only a trend toward elevated TNF- $\alpha$ and IL- 6 serum levels in middle-aged and old mice relative to levels in young mice (Supplemental Figure 4A), both of these cytokines were consistently and significantly increased in the BM of aged mice (Figure 3, C and D). Furthermore, both IPA (Figure $3 \mathrm{E}$ ) and GSEA (Figure 3F) revealed that the expression of TNF- $\alpha$ and its target genes was significantly increased in aged B cell progenitor populations $\left(P=4.23 \times 10^{-5}\right.$ for TNF- $\alpha$ stimulatory pathways using IPA). These findings were corroborated by flow cytometric analyses, which revealed that the production of TNF- $\alpha$ significantly increased with age in pro-B cell progenitors (Supplemental Figure $4 \mathrm{~B})$. In addition, BM stromal cell cultures from old mice exhibited elevated production of IL- $1 \beta$ and IL- 6 relative to the levels detected in cultures from young mice, while BM B cell lineage cultures from old mice only exhibited increased IL-6 production (Supplemental Figure 4, C-E). In addition to increased Tnfa mRNA expression, Ifnz and IFN- $\alpha$-inducible protein 27 (Ifi27) mRNA expression levels were also evident in sorted pro-B cell progenitors isolated from old mice (Figure 3G). Similarly, Muc5b, which has been shown to be activated in a variety of cell types in response to inflammation (43), was only found to be expressed in aged, but not young, pro-B cell progenitors. These experiments revealed that aging is associated with increased inflammation in the BM and within resident $\mathrm{B}$ cell progenitors. Contrasting with the correction of aging-associated fitness defects in pro-B cells (Figure 2), the expression of $B C R-A B L$ and $N R A S^{V 12}$ significantly increased the expression of Tnfa in aged pro-B cell progenitors (Figure $3 \mathrm{H}$ ). Notably, the expression of $B C R$ $A B L, N R A S^{V 12}$, or $M y c$ did not elicit TNF- $\alpha$ production from young pro-B cell progenitors (Figure $3 \mathrm{H}$ ). Thus, oncogene expression can actually exacerbate the expression of inflammatory cytokines specifically in old progenitors.

Ectopically promoting inflammation in young mice phenocopies aging-associated $B$ lymphopoiesis. We asked whether increasing inflammation is sufficient to impair B cell progenitor fitness. To this end, young mice were injected with LPS or recombinant TNF- $\alpha$ every 4 days for a period of 2 weeks. As a control, we injected $\alpha \mathrm{IL}-7$ to impair B lymphopoiesis. After 2 weeks of treatment, we observed significant reductions in the percentages of pro-, pre-, and immature B cells in the BM of mice treated with $\alpha \mathrm{IL}-7$ or inflammatory mediators (LPS or TNF- $\alpha$ ) (Figure $4 \mathrm{~A}$ ), consistent with previous results (22-24). Importantly, acute inflammation also significantly decreased expression levels of the $\mathrm{B}$ lineage specification genes Pax5 and EbF (Figure 4B) as well as expression of the purine synthesis genes Hprt and Gmps (Figure $4 \mathrm{C}$ ) in B cell progenitors, providing molecular insight into reduced $\mathrm{B}$ progenitor fitness with old age. Collectively, these data demonstrate that induction of inflammation in young mice mirrors the $\mathrm{B}$ lymphopoiesis defects observed in old mice.

Aging in most vertebrates is commonly associated with lowgrade, chronic inflammation (31). We investigated whether the chronic inflammation that we observed in old mice can underlie their B lymphopoiesis defects. We monitored B cell development in TNF- $\alpha^{\triangle A R E}$ mice, which have a mutation in the Tnfa $3^{\prime}-\mathrm{UTR}$, removing regulatory adenylate-uridylate-rich (AU-rich) elements and leading to increased constitutive TNF- $\alpha$ expression. These mice exhibit heightened susceptibility to chronic inflammatory diseases such as rheumatoid arthritis and Crohn disease $(44,45)$. Young TNF- $\alpha^{\triangle A R E}$ mice had significantly ( $\sim 5$-fold) increased circulating TNF- $\alpha$ levels (Figure 4D). B lymphopoiesis was significantly reduced in young TNF- $\alpha^{\triangle A R E}$ mice, coinciding with an increased frequency of myeloid cells (Figure 4, E and F). Furthermore, 
A PCA mapping $91.5 \%$

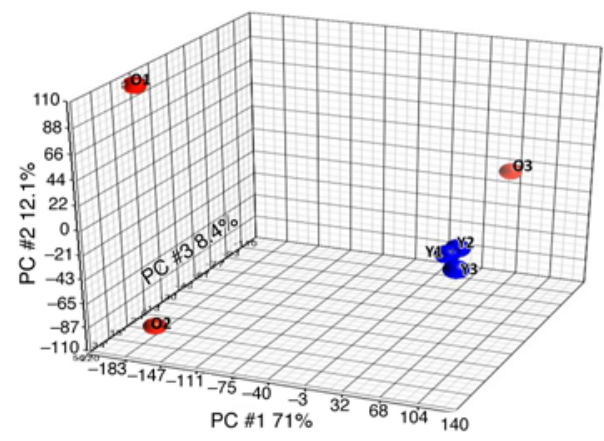

B

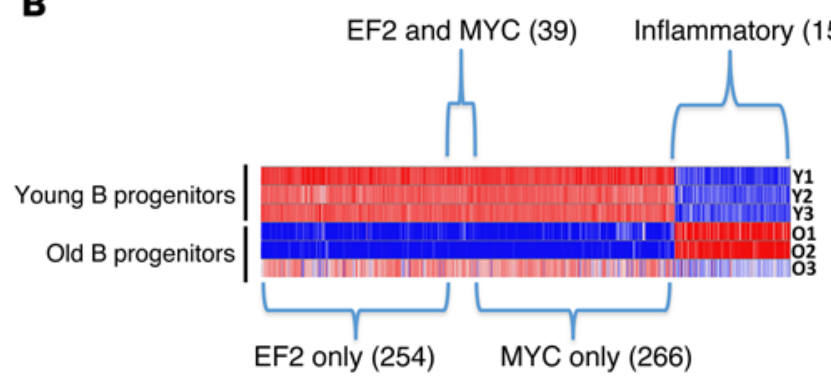

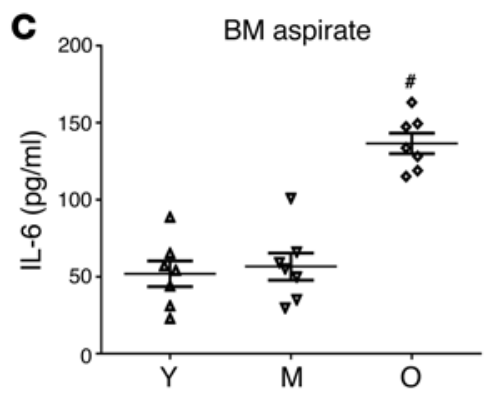

$\mathbf{E}$
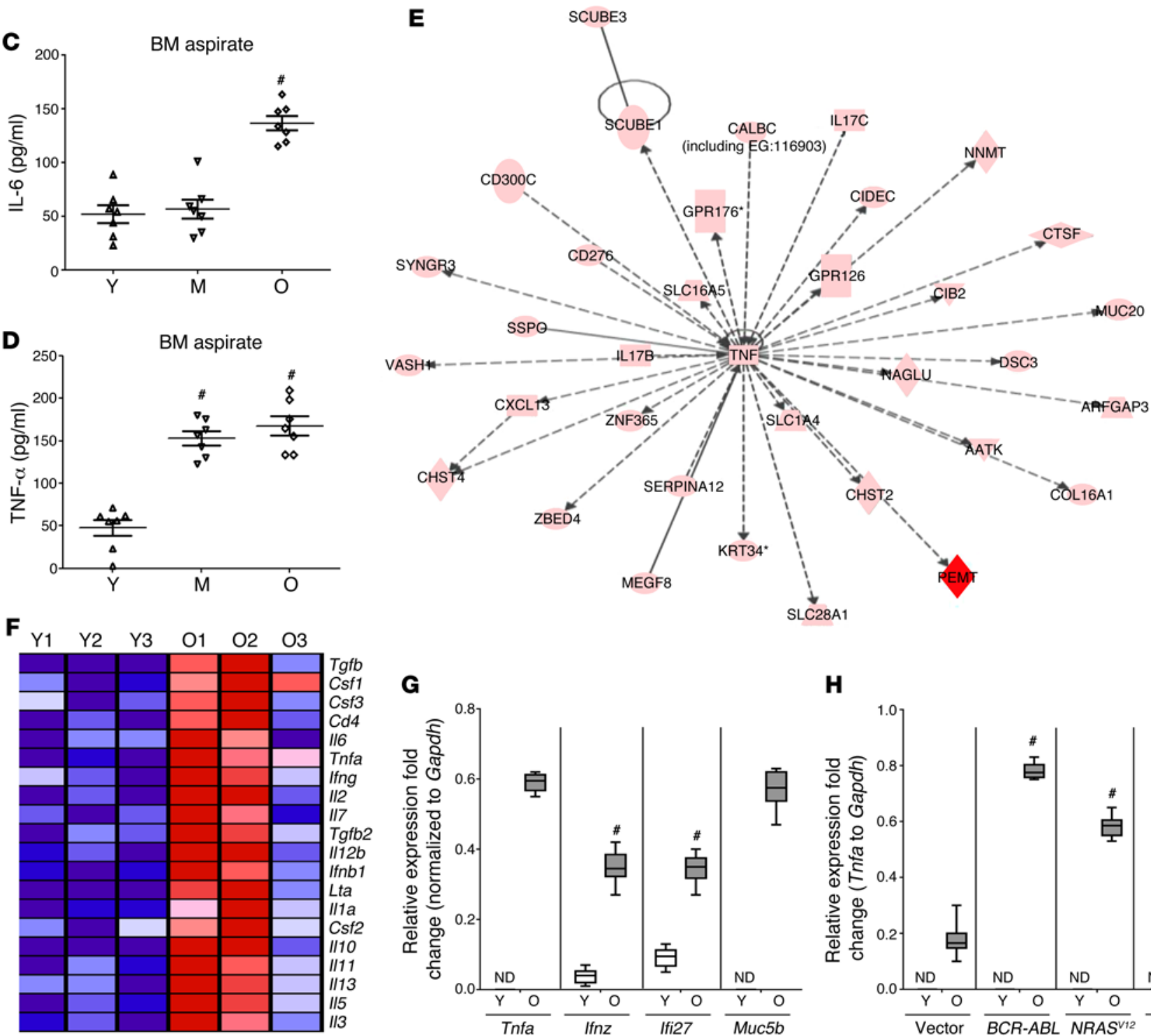

G

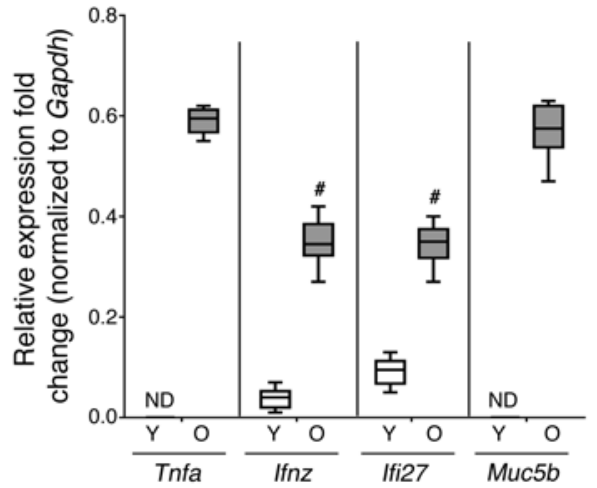

H

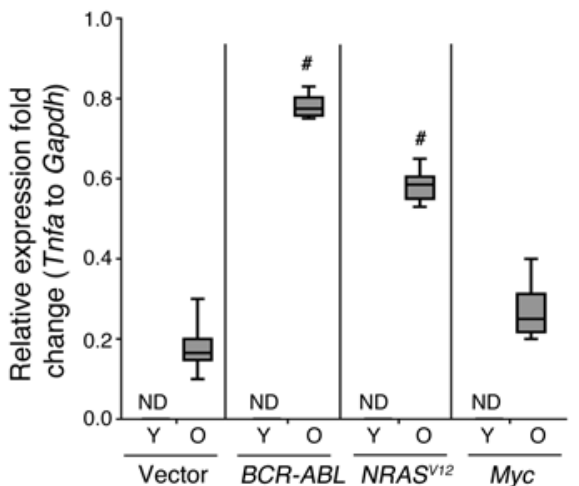

Figure 3. Increased inflammation in the BM with age coincides with decreased expression of genes regulating cell-cycle progression in B progenitors. (A) PCA of the microarray data was generated using the Partek Genomics Suite. (B) Gene expression profiles from the microarray analysis performed on $\mathrm{B}$ cell progenitors isolated from young and old mice were analyzed by CSEA for the expression of genes regulated by E2F and MYC and of those involved in inflammatory processes. (C and D) BM aspirates were collected from young ( $Y$; 2-month-old), middle-aged (M; 14-month-old), and old (0; 24-month-old) mice, and IL-6 (C) and TNF- $\alpha$ (D) levels were determined using ELISA. Values represent the mean \pm SEM of 2 independent experiments, with more than 6 mice per age group. (E) Network analysis of the microarray data was performed using IPA software, which identified TNF- $\alpha$ as an important cytokine that increases in aged B progenitors. (F) Heatmap showing a subset of the inflammatory genes shown in B. (C) Pro-B cell progenitors from young (2-monthold) and old (24-month-old) mice were sorted, and expression levels of inflammatory genes (Tnfa, Ifnz, and Ifi27) or of those regulated by inflammation (e.g., Muc5b) were determined by qPCR. (H) Expression levels of TNF- $\alpha$ in sorted young or old pro-B cells expressing vector or oncogenic BCR-ABL, NRASV12, or Myc (all GFP') were determined using qPCR. Values in $\mathbf{G}$ and $\mathbf{H}$ represent the mean \pm SEM of 4 independent experiments, with more than 10 mice per age group. ${ }^{\#} P<0.001$, by Student's $t$ test relative to young controls. ND, not determined. 

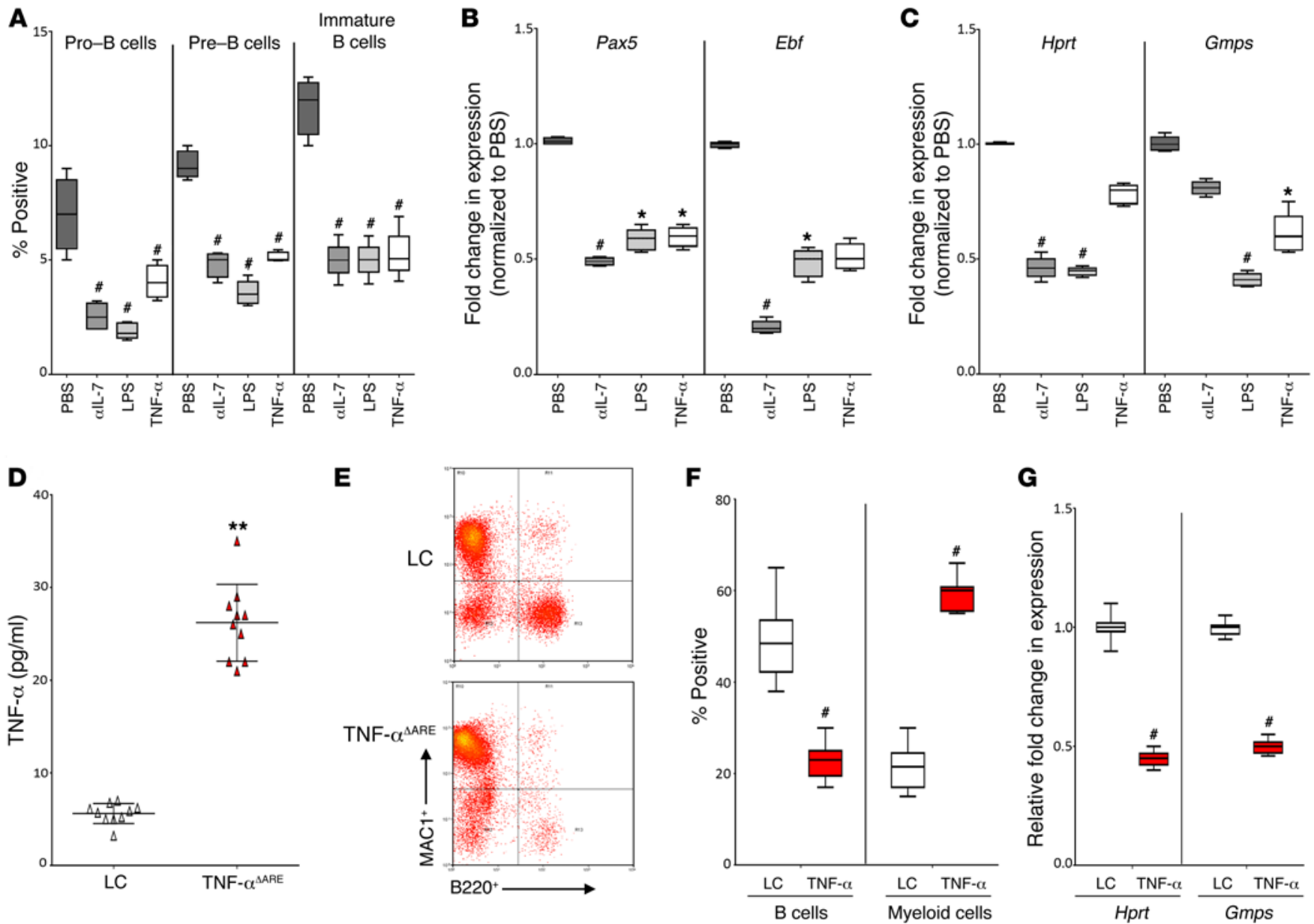

Figure 4. Induction of inflammation promotes functional decline in B progenitors. (A-C) Young mice were injected every 4 days for 2 weeks with PBS (vehicle), $\alpha$ lL-7-neutralizing Abs, LPS, or recombinant TNF- $\alpha$. After 2 weeks of treatment ( 3 injections total), the mice were sacrificed and the percentage of $B$ progenitors in the BM determined using flow cytometry. Expression levels of B lineage-specification genes (B) and purine synthesis genes (C) were determined using qPCR. Values in A-C represent the mean \pm SEM for 5 mice per treatment group. (D-C) Serum TNF- $\alpha$ levels in 5-month-old TNF- $\alpha^{\triangle A R E}$ mice and their littermate controls were measured by ELISA (D), frequencies of B cell (B220+) and myeloid (MAC1+) progenitor cells in BM were determined by flow cytometry (E and $\mathbf{F}$ ), and mRNA levels of purine synthesis genes in pro-B cells were determined by qPCR (C). Values represent the mean \pm SEM of 3 independent experiments, with more than 9 mice per group. ${ }^{*} P<0.05,{ }^{* *} P<0.01$, and ${ }^{\#} P<0.001$, by Student's $t$ test relative to PBS-injected mice or littermate controls. LC, littermate control.

chronic TNF- $\alpha$ exposure significantly reduced Hprt and Gmps gene expression levels in sorted pro-B cells (Figure $4 \mathrm{G}$ ). We conclude that chronic TNF- $\alpha$ exposure in young mice impairs the fitness of pro-B cells in a manner similar to that observed with aging.

Reducing inflammation prevents aging-associated fitness defects in $B$ cell progenitors. We used transgenic mice that express either human AAT or human IL-37 to determine whether either of these antiinflammatory mediators could protect against age-associated fitness reductions in B progenitors. AAT is a serum serine protease inhibitor with potent antiinflammatory activity. Transgenic expression of human AAT under the surfactant protein $C$ promoter results in low circulating levels of AAT $(46,47)$. Nevertheless, mice transgenic for AAT (AATtg) exhibit a remarkable resistance to a broad spectrum of inflammatory and immunological challenges (48). IL-37 is an antiinflammatory member of the IL-1 family that broadly inhibits innate inflammation (49). IL-37 transgenic (IL-37tg) mice expressing human IL-37 are protected in models of endotoxin shock, colitis, and ischemia reperfusion injury (50,
51). Strikingly, the aging-associated increases in TNF- $\alpha$, IL-6, and IL-1 $\beta$ levels in the BM and serum of 20-month-old mice were prevented by transgenic expression of either AAT or IL-37 (Figure 5).

We observed significant reductions in the number of pro-, pre-, and immature B cell populations in aged AATtg (Figure 6A) and IL-37tg (Figure 6D) mice that were comparable to the reductions seen in nontransgenic controls. However, pro-B cells from older AATtg or IL-37tg mice had STAT5 activation levels similar to those observed in young mice, contrasting with cells from older nontransgenic mice (Figure 6, B and E). Furthermore, AAT and IL-37 expression prevented the aging-associated reductions in Hprt, Gmps, and Myc gene expression in aged pro-B cells (Figure $6, \mathrm{C}$ and F). AAT expression also decreased the proinflammatory state of aged pro-B cells, as indicated by significant decreases in the expression levels of TNF- $\alpha$ and IFNip27. Combined, these data suggest that aged, antiinflammatory microenvironments preserve the fitness of $\mathrm{B}$ cell progenitors, while failing to prevent the aging-associated reductions in B progenitor frequency. 

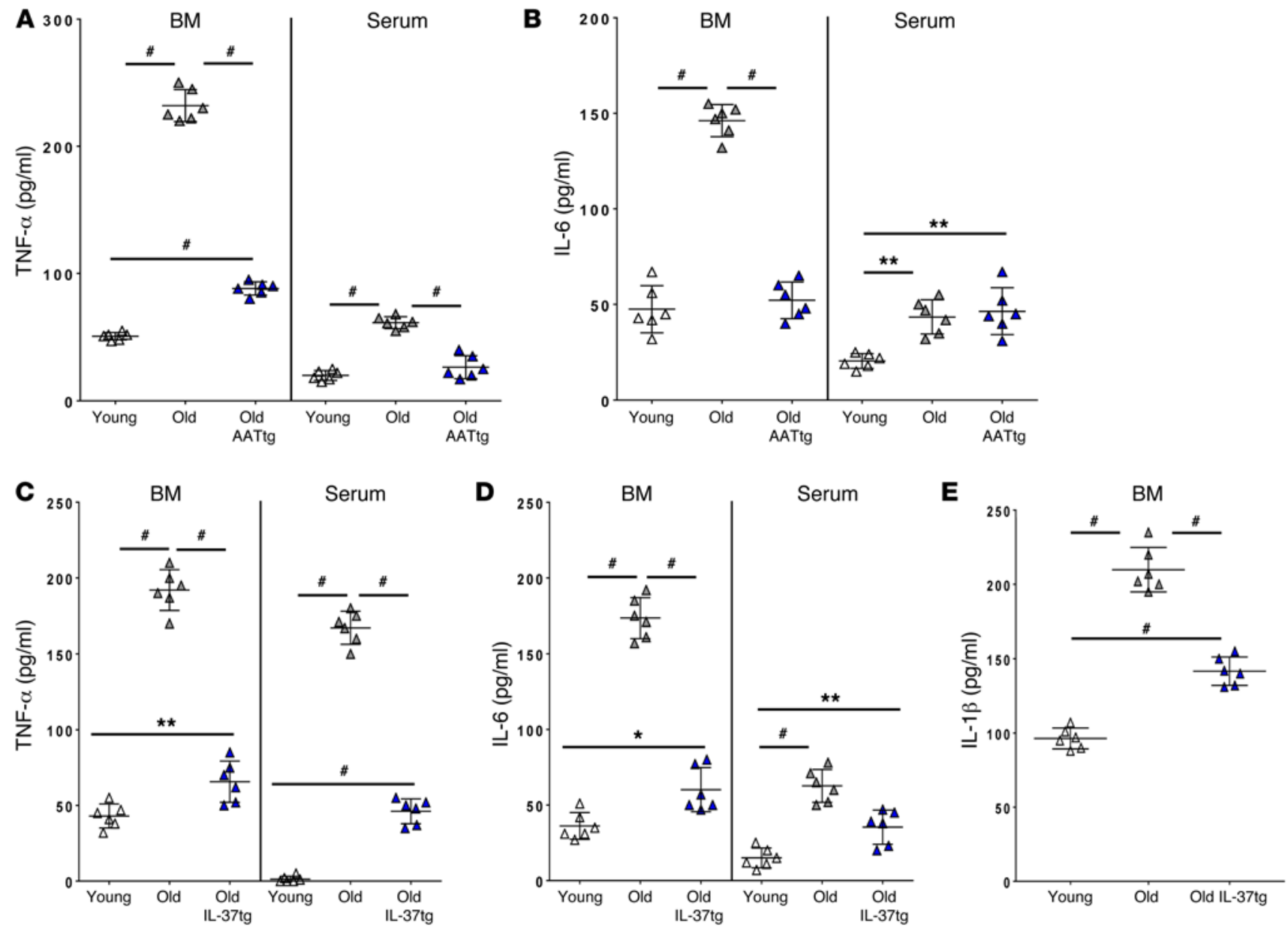

Figure 5. Proinflammatory cytokines are reduced in old antiinflammatory transgenic mice. ELISAs for TNF- $\alpha$, IL-6, and IL-1 $\beta$ were performed on BM aspirates and serum collected from young (2-month-old) and old (20-month-old) littermates, old AATtg mice (A and B), and old IL-37tg mice (C-E). Values represent the mean \pm SEM of 2 independent experiments ( 6 mice total). ${ }^{*} P<0.05,{ }^{* *} P<0.01$, and ${ }^{\#} P<0.001$, by Student's $t$ test relative to young controls.

Reducing inflammation in aged backgrounds prevents selection for oncogenic NRAS $S^{112}$. We next determined whether reducing inflammation in aged mice altered the selection for oncogenically initiated cells. Young and old AATtg mice and age-matched controls were injected with the same pool of young BM progenitor cells transduced with retroviral vectors expressing NRAS $S^{V 12}$ plus GFP or cyan fluorescent protein (CFP) only (Figure 7A). Thus, it is the age and inflammatory status of the host microenvironment, not the cells expressing the oncogene or vector control, that is being varied. Notably, expression of the control CFP vector by peripheral B cells was not influenced by the host's age or genetic background and did not exhibit significant changes over time (Figure 7B), indicating that homing of transduced cells and their maintenance in the host (including potential immune rejection) were not impacted by the recipient mouse's age or AAT transgene expression. In striking contrast, we observed clear and exclusive selection for $N R A S^{V 12}$ expression in the $\mathrm{B}$ cell lineage in old recipients, but not in young or old AATtg recipients (Figure 7C), which mirrored the selection for NRAS ${ }^{V 12}$ expression in BM B progenitors only in the nontransgenic old recipients (Figure 7D). Thus, selection for NRAS $S^{V 12}$ expression was completely prevented in B progenitor pools in the aged antiinflammatory AATtg backgrounds, indi- cating that aging-related inflammation is a key driver of oncogenic adaptation in B progenitors.

STAT5 activity is reduced in host $\left(\mathrm{CD} 45.2^{+}\right)$pro-B cells isolated from old mice, and this decline was prevented in host pro-B cells isolated from aged AATtg mice (Figure 7E). We asked whether the age and inflammatory status of the host had an impact on fitness parameters for $\mathrm{B}$ progenitors that developed from transplanted young $\mathrm{BM}\left(\mathrm{CD} 45.1^{+}\right)$. Indeed, young $\mathrm{CD} 45.1^{+}$pro-B cell progenitors that developed in aged recipient mice also exhibited significantly reduced STAT5 activation, but not if the recipients were old AATtg mice (Figure 7F; donor $\mathrm{CFP}^{+}$cells). Similarly, expression levels of Hprt, Gmps, and Stat $5 b$ were all reduced in the young donor pro-B cells that developed in aged recipient mice, but not if the recipients were AATtg or young (Figure 7, G and H and Supplemental Figure 5B). Thus, these key fitness parameters for B progenitors are dictated by the age and inflammatory status of the host microenvironment. Strikingly, $N R A S^{V 12}$ expression restored p-STAT5 activation and mRNA levels of Hprt, Gmps, Myc, and Stat $5 b$ in pro-B cells in aged recipients to the levels observed in young recipients (Figure 7F; donor $\mathrm{GFP}^{+}$cells), providing a rationale for NRAS activation-dependent adaptation in the aged/inflammatory background. Notably, ERK activation in B progenitor cells was not affected by 

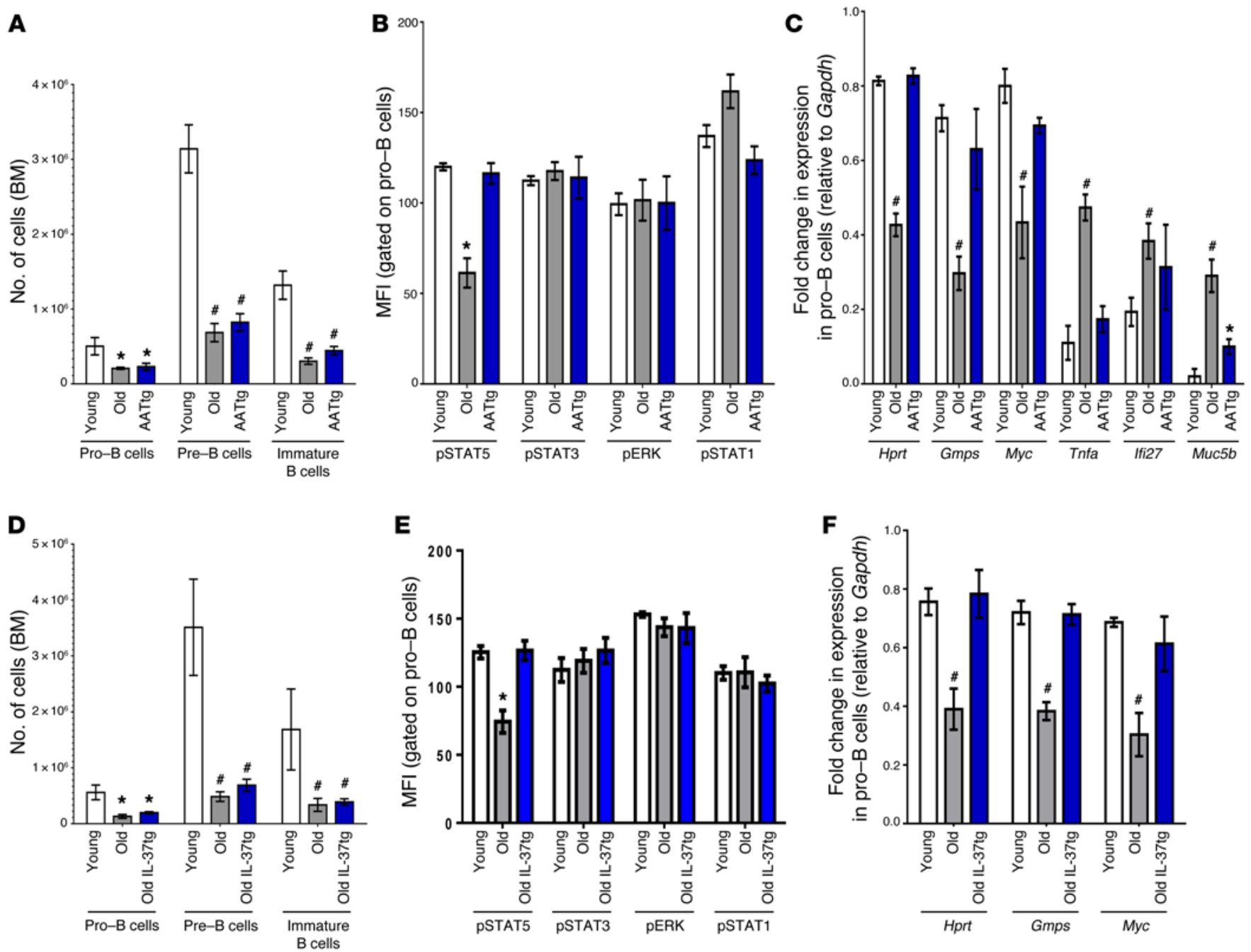

Figure 6. Reducing inflammation prevents declines in aging-associated B progenitor fitness. BM from young (2-month-old) and old (20-month-old) littermate and AATtg and IL-37tg mice was analyzed for the frequency of B cell progenitor populations (A and D). Activation of STAT5, STAT3, ERK, and STAT1 in pro-B cells was determined by flow cytometry ( $B$ and $\mathbf{E}$ ). mRNA expression of genes involved in cell-cycle regulation and inflammation in sorted pro-B cells was determined by qPCR ( $\mathbf{C}$ and $\mathbf{F}$ ). Values represent the mean \pm SEM of 2 independent experiments, with more than 5 mice per group. ${ }^{*} P<0.05$ and ${ }^{\#} P<0.001$, by Student's $t$ test relative to young controls for each experiment.

the microenvironment in which the cells developed (Supplemental Figure 5A). AAT also prevented age-dependent increases in the expression of the inflammatory mediators TNF- $\alpha$, IFNip27, and MUC5B in B progenitors (Figure 7H and Supplemental Figure 5B). Note that we were unable to analyze $N R A S^{V 12}$-initiated pro-B cells in the young or AATtg backgrounds, as these cells were not detectable above background levels (Figure 7D).

To corroborate these results using an alternative means to dampen age-dependent inflammation, we used IL-37tg mice. Similar to the above-described methods, young and old IL-37tg mice and age-matched controls were injected with the same pool of young $\mathrm{BM}$ progenitor cells transduced with retroviral vectors expressing $N R A S^{V 12}$ plus GFP or CFP only. Expression of IL-37 in aged mice also prevented the selection of oncogenic $N R A S^{V 12}$-expressing $\mathrm{B}$ progenitor cells (Figure 8A). Selection for NRAS ${ }^{V 12}$ was not observed in myeloid lineage fractions (Supplemental Figure 5C). Similar to findings in old AATtg recipients, young pro-B cell progenitors that developed in aged IL-37tg mice exhibited STAT5 activation and
Hprt and Gmps gene expression levels similar to those detected in pro-B cells in young recipients, and NRAS ${ }^{V 12}$ expression prevented reductions in these fitness parameters in pro-B cells that developed in aged littermate control mice (Figure 8, B and C). In all, our experiments using both AATtg and IL-37tg models indicate that key fitness parameters of B cell progenitors can be dictated by the state of the microenvironment (age and inflammatory status) and that NRAS activation can rescue defects that result from aged/inflammatory microenvironments, facilitating clonal expansion of oncogenically initiated B progenitors.

\section{Discussion}

Aging is the single most important prognostic factor associated with the development of many diseases including cancer (1). The results described here demonstrate that aging-associated increases in inflammation negatively impact the development and function of B progenitor cells, including reductions in key indicators of cell fitness. Importantly, using two different, natu- 
A
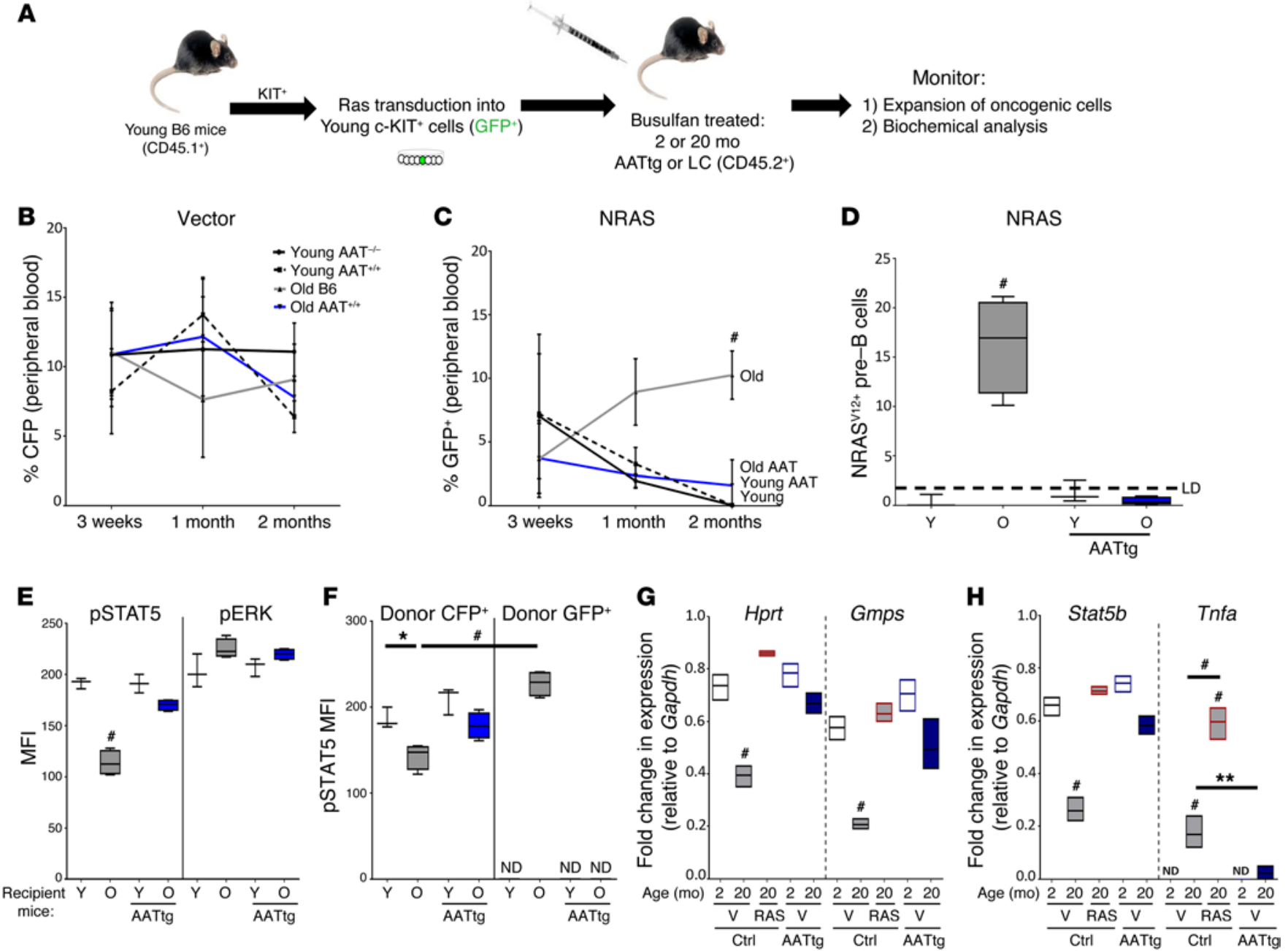

Figure 7. Reducing inflammation in aged AATtg mice suppresses selection for oncogenic NRAS ${ }^{v 12}$-expressing B progenitors. (A) Experimental overview. (B and C) Frequencies of young, vector-expressing (CFP+) cells (B) or young, NRAS ${ }^{12}$-expressing (GFP+) cells (C) in the peripheral blood of recipient mice were monitored for 2 months after transplantation. (D) After 2 months, mice were sacrificed, and the frequencies of NRAS ${ }^{V 12}$-expressing pre-B cell progenitors in the BM of recipient mice were determined by flow cytometry. LD, limit of detection. (E) Recipient pro-B cells (CD45.2+) were analyzed by flow cytometry for STAT5 and ERK activation. (F) STAT5 activation in donor pro-B cells (CD45.1+) expressing vector (CFP+) or NRAS ${ }^{12}$ (GFP+) was analyzed using flow cytometry. (G and $\mathbf{H})$ Expression levels of the indicated genes in sorted donor (CD45.1 $)$ pro-B cells expressing vector (V) or NRAS ${ }^{122}$ (RAS) were determined by qPCR . Values in B-H represent the mean \pm SEM, with more than 5 mice per group. (B-E and $\mathbf{G}){ }^{\#} P<0.001$, by Student's $t$ test relative to young controls. ( $\mathbf{F}$ and $\mathbf{H}){ }^{*} P<0.05,{ }^{* *} P<0.01$, and ${ }^{*} P<0.001$, by 1 -way ANOVA. Ctrl, control.

rally occurring molecules to reduce inflammation in old mice, we show that preventing aging-associated reductions in B progenitor fitness abrogates selection for NRAS ${ }^{V 12}$-initiated progenitors. These studies highlight how inflammation-induced alterations in the adaptive landscape in old age govern the selection of oncogenically initiated cells and, ultimately, leukemogenesis within hematopoietic progenitor cell populations (Figure 8D).

In recent years, there has been growing interest in identifying factors that regulate the aging process $(8,52,53)$. We observed aging-associated increases in TNF- $\alpha$, IL- 6 , and IL- $1 \beta$ levels in the $\mathrm{BM}$ of old mice. Previous studies demonstrate that aged BM stromal cells and mature B cells that accumulate in the BM are two sources of inflammation that manifest with age $(25,54)$. In this study, we further demonstrated that aged pro B cell progenitors also produce proinflammatory cytokines such as TNF- $\alpha$. Notably, in addition to providing resistance to the effects of inflammatory cytokines on B progenitor fitness in aged backgrounds, $B C R-A B L$ and oncogenic $N R A S^{V 12}$ expression is associated with increased expression of inflammatory cytokines in old pro-B cells, perhaps further impairing the fitness of competing nononcogeneexpressing progenitors (consistent with previous results in HSCs, refs. 55-57). We observed that either acute or chronic inflammation was sufficient to suppress key factors regulating normal B cell development (Pax5 and Ebf) and function (Hprt and Gmps), resulting in significant decreases in B lymphopoiesis and phenocopying the aging process. From these studies, we conclude that inflammatory mediators negatively regulate B lymphopoiesis and accelerate reductions in B progenitor fitness, suggesting that chronic inflammation promotes B progenitor fitness impairments that manifest in old age.

Heterochronic parabiosis experiments in mice indicate that the aging process, for some organs and tissues, is substantially 
A

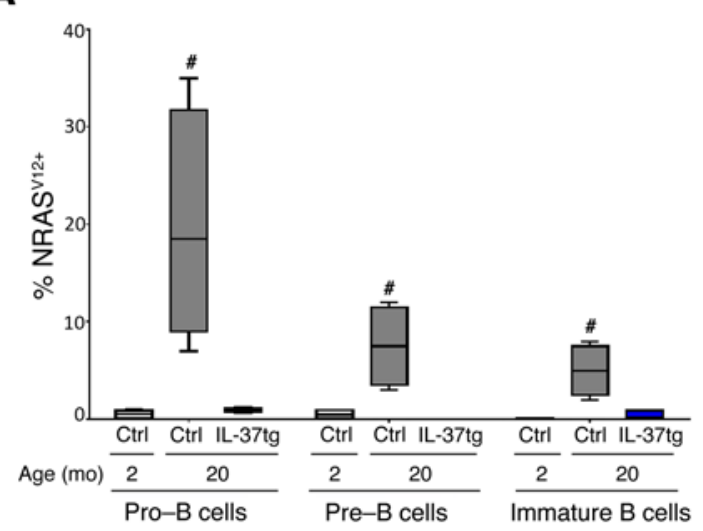

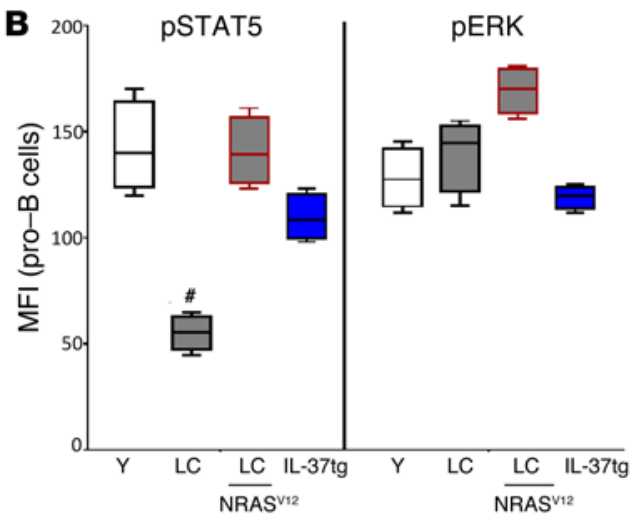

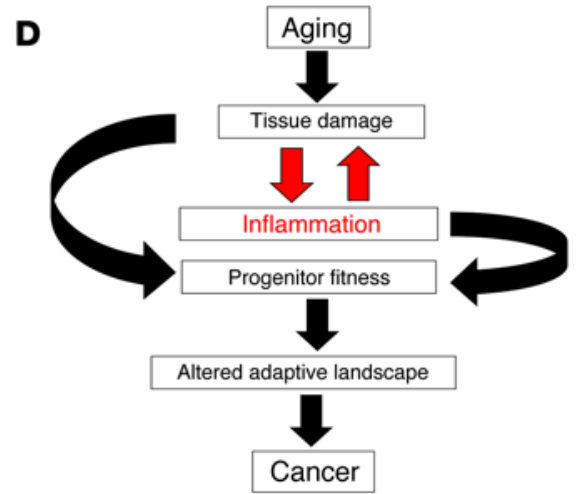

Cancer

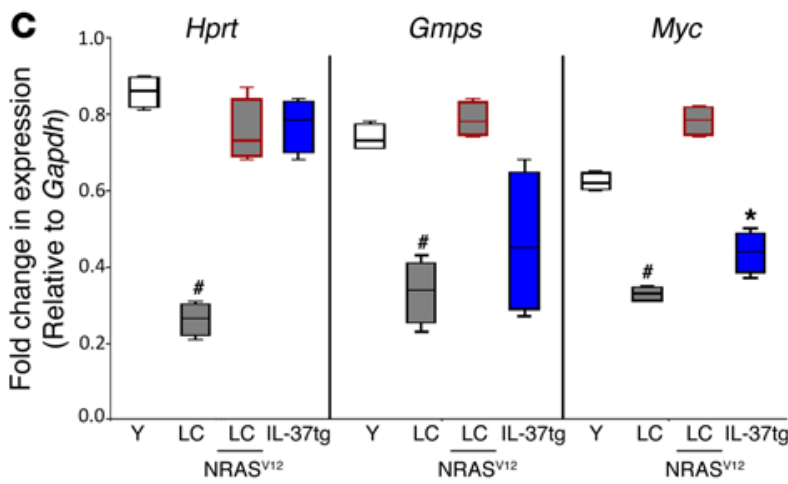

Figure 8. Reducing inflammation in aged IL-37tg mice abrogates NRAS ${ }^{12}$-mediated oncogenesis. Young and old (2-month-old and 20-month-old, respectively) littermate (LC) or IL-37tg mice were transplanted with young NRAS ${ }^{\mathrm{V} 12}$-expressing cells, as in Figure 7A. Three months after transplantation, BM was analyzed by flow cytometry for (A) the frequency of NRAS ${ }^{12}$-expressing cells in B progenitor cell populations and (B) activation of STAT5 and ERK in pro-B cells expressing NRAS ${ }^{122}$ (red-outlined gray boxes) or not expressing the oncogene. (C) mRNA levels of Hprt, Gmps, and Myc in sorted pro-B cells were determined by qPCR (note: NRAS ${ }^{v 12}$-initiated cells only expanded in the old littermate control mice). (D) Model for how aging and aging-associated inflammation regulate progenitor cell fitness and oncogenesis. Values in $\mathbf{A}-\mathbf{C}$ represent the mean $\pm \mathrm{SEM}$, with more than 5 mice per group. ${ }^{*} P<0.05$ and ${ }^{\#} P<0.001$, by Student's $t$ test relative to young controls.

influenced by non-cell-autonomous factors $(52,53)$. Previous studies have shown that both cell autonomous and non-cellautonomous (microenvironmental) factors contribute to hematopoietic aging, including functional changes in HSCs and B lymphopoiesis $(40,58,59)$. Indeed, we show that young BM progenitors transplanted into old mice (mildly conditioned with busulfan) produce $\mathrm{B}$ progenitors with signaling defects that mirror those of old progenitors, unless the old recipient mice express antiinflammatory mediators. Thus, the age and inflammatory status of the host mouse dictates the fitness of $\mathrm{B}$ progenitors produced by young HSCs in the host BM microenvironment. Nonetheless, our results also demonstrate that $\mathrm{B}$ lymphopoiesis derived from old HSCs transplanted into young mice still maintains aging-associated fitness defects, as indicated by substantial reductions in IL-7mediated signaling and the expression of key metabolic and DNA replication genes. The inability of a young microenvironment to reverse aging-associated defects in hematopoiesis may result from substantial aging-associated genetic and epigenetic changes in HSPC populations $(39,60-62)$, even when caused by cellextrinsic factors such as inflammation.

Focusing on inflammation, we asked how age-dependent changes in the microenvironment could alter the fitness of B cell progenitors. Interestingly, we still observed a marked decline in B progenitor numbers in aged AATtg and IL-37tg mice. These results suggest that this decline in $B$ lymphopoiesis may be an evolved program that reduces $B$ cell production once a diverse $B$ cell repertoire has developed during youth. The aging-associated biasing of hematopoiesis toward myelopoiesis may be advantageous in order to better combat infections and repair tissue injury. Nonetheless, we found that both AAT and IL-37 expression prevented functional impairment in IL-7R signaling and the expression of purine synthesis genes in pro- $\mathrm{B}$ cell progenitors. Therefore, the late-life decline in the function of HSPCs (reduced fitness on a per-cell basis) can, in good measure, be explained by inflammation that increases late in life, beyond the period when reproduction is likely and thus largely outside the influence of natural selection.

In our mouse models, we observed selection for the tested oncogenic mutations within old B progenitor populations, leading to the development of B-lineage leukemias. This selection was not observed in young hematopoietic backgrounds due to the inability of oncogenic mutations to significantly improve the fitness of young B progenitor cells (thus, the highly fit B progenitors in young microenvironments provide effective competition 
to limit the expansion of oncogenically initiated progenitors). Notably, hematopoietic malignancies of the B lineage predominate in older mice, while in elderly humans, both B lymphoid and myeloid leukemias are common $(63,64)$. B lymphoid leukemias are also common in young children, and infections and the associated inflammation have been proposed to play a role in their development (65). Whether in humans or mice, contexts that lead to reduced fitness of stem/progenitor cells would be expected to lead to selection for adaptive oncogenic events in these stem and progenitor cell populations and to malignancies of correspondingly different types.

Inflammation has been associated with the progression of many solid tumors. Its actions in promoting tumorigenesis have largely been attributed to the ability of inflammatory cells to create an immune-suppressive microenvironment, promote metastasis, and enhance the protumorigenic phenotypes of cancer cells including proliferation, survival, and invasion $(66,67)$. Notably, our studies indicate that inflammation can promote selection for oncogenic events in HSPCs by suppressing overall progenitor fitness, providing a context that favors oncogenic adaptation. These findings complement emerging studies that have shown increased hematopoietic clonal evolution (including for clones with known oncogenic drivers) in the elderly (68-72). Inflammation has also been proposed to contribute to the genesis of myeloproliferative disorders that are almost exclusively present in the elderly $(73,74)$, and increased oncogenic clonal expansions and clonal hematopoiesis have also been demonstrated in old mice $(75,76)$. Notably, the incidence of leukemias is higher in individuals with chronic inflammatory disease (77), although the cause of this association is unknown. Moreover, prophylactic aspirin use has been shown to reduce the incidence of colorectal cancer, breast cancer, and leukemias (78-80). Interestingly, polymorphisms associated with reduced inflammation are preferentially found in centenarians $(81,82)$.

Importantly, our studies provide a potential explanation for the mechanism underlying increased oncogenic clonal evolution and leukemias in old age. We demonstrated that the oncogenes $B C R-A B L, N R A S$, and $M y c$ can reverse aging-associated functional defects in $B$ progenitors, promoting selection for progenitors expressing these oncogenes specifically in the aged hematopoietic context. These oncogenes do not restore differentiation and likely convey other transformed phenotypes to the recipient cells. However, in the BM microenvironment of antiinflammatory aged mice, selection for $N R A S^{V 12}$ was completely suppressed. We propose that the maintenance of hematopoietic progenitor fitness in both young and antiinflammatory, aged backgrounds can prevent oncogenic adaptation via stabilizing selection $(35,83)$. While oncogenic NRAS can restore fitness parameters (such as for signaling) in B progenitors (whether young or old) in an old hematopoietic background, it does not do so for progenitors in either young microenvironments or old but antiinflammatory microenvironments, as there appears to be little room for improvement.

In conclusion, our study demonstrates that aging is characterized by reductions in key parameters of B progenitor cell fitness and increased selection for oncogenic events, both of which are substantially modulated by the degree of aging-associated inflam- mation. Overall our study indicates that dampening inflammation in older populations may reduce aging-associated functional impairment in hematopoiesis, which in turn may reduce leukemia incidence in these populations by preventing the selection for oncogenically initiated cells.

\section{Methods}

Microarray data were deposited in the NCBI's Gene Expression Omnibus (GEO) database (GEO GSE67827). Microarray, mass spectrometry, and NMR methods are provided in the Supplemental Methods.

\section{Mice}

BALB/c and C57BL/6 mice of different ages were purchased from the National Institute of Aging (NIA) or the National Cancer Institute (NCI). CD45.1 congenic mice (B6.SJL-Ptprc ${ }^{\mathrm{a} P e p c}$ /BoyCrCrl) were purchased from Charles River Laboratories. TNF- $\alpha^{\triangle \mathrm{ARE}}$, AAT, and IL-37 transgenic mice were backcrossed onto a C57BL/6 background for more than 10 generations, and both transgene-positive and transgene-negative littermates were aged in-house. Male and female mice from each strain were used in these studies.

\section{Retroviral transduction and BM transplantation}

Retroviral transductions and BM transplantations were performed as previously described (84). The retroviral murine stem cell virusires-GFP (MSCV-ires-GFP) (MiG), MiG-BCR-ABL, MiG-RAS, MiG$\mathrm{Myc}$, and MSCV-ires-CFP (MiC) vectors were used to introduce the respective genes into MACS-purified (Miltenyi Biotec) murine c-KIT ${ }^{+}$ hematopoietic progenitor cells. These cells were transduced with $\mathrm{MiG}$ viruses in nonadhesive 6-well plates by incubation at $37^{\circ} \mathrm{C}$ for 2 hours in the presence $8 \mu \mathrm{g} / \mathrm{ml}$ polybrene and $20 \mu \mathrm{g} / \mathrm{ml}$ stem cell factor, followed by the spin-fection technique (centrifugation at $910 \mathrm{~g}$ for 2 hours). Cells were then washed once with $1 \times$ PBS and transplanted into conditioned recipient mice.

Mice were condition using sublethal irradiation (5 Gy), lethal irradiation (10 Gy split dose), or busulfan $(25 \mathrm{mg} / \mathrm{kg})$ and injected with transduced cells 2-4 days after treatment (depending on the conditioning protocol). In experiments in which recipient mice were lethally irradiated (Figure 2, G and $\mathrm{H}$ ), mice were injected with $2 \times 10^{6}$ unsorted BM cells from young (2-month-old) or aged (22- to 24-month-old) donor mice (prepopulated with young or aged competitors, respectively). Before lethal irradiation, mice were deprived of food overnight to reduce small intestinal cytotoxicity. Four days after prepopulation, $2 \times 10^{5}$ MiG-transduced c-KIT ${ }^{+}$hematopoietic progenitor cells were then transplanted i.v. into recipient mice.

\section{Inflammation-inducing and IL-7-neutralizing experiments}

IL-7 neutralization experiments were performed as previously described (16). In these experiments, IL-7-neutralizing Abs were injected at a dose of $0.5 \mathrm{mg} / \mathrm{mouse}$ every 4 days until the experiment was terminated. In the experiments described in Figure 4, LPS was injected at a dose of $1 \mathrm{mg} / \mathrm{kg}$, and recombinant TNF- $\alpha$ was injected at a dose of $5 \mu \mathrm{g} /$ mouse every 4 days until the experiment was terminated.

\section{EdU analysis of B progenitor populations}

EdU analysis of B cell progenitor populations was performed using the Click-iT EdU Alexa Fluor 647 Imaging Kit (Life Technologies; catalog C10634). C57BL/6 mice were injected i.p. with EdU (2 mg/mouse, 
dissolved in PBS) for 2 hours. After 2 hours, mice were sacrificed, and $10^{6} \mathrm{BM}$ cells were collected and surface stained to identify pro-, pre-, and immature $\mathrm{B}$ cell progenitor populations. After staining, cells were washed once with $1 \times$ PBS and the pellets resuspended in $100 \mu$ l ClickiT fixative (Life Technologies) for 15 minutes at room temperature. After fixation, cells were again washed and incubated for 10 minutes in $50 \mu$ l diluted Click-iT saponin-based permeabilization solution (Life Technologies). After the 10-minute incubation period, $250 \mu$ reaction mix (including $1 \times \mathrm{PBS}, \mathrm{CuSO}_{4}$, Alexa Fluor 647, and reaction buffer additive, combined following the manufacturer's protocol) was added to each sample and incubated for 30 minutes at room temperature. Next, cells were washed and resuspended in $1 \times$ PBS containing $50 \mu \mathrm{g} /$ $\mathrm{ml}$ PI (Roche). Samples were run on a Gallios cytometer (Beckman Coulter). The normalized $\mathrm{x}$-mean mean fluorescence intensity (MFI) of $\mathrm{EdU}^{+}$cell populations (which is a measure of S-phase progression) was calculated using the following formula: [(x-mean MFI of EdU $\mathrm{U}^{+}$ cells) - (x-mean MFI of the $\mathrm{G}_{1}$ population)].

\section{Flow cytometric analysis}

Surface staining. Single-cell suspensions were plated in 96-well round-bottomed plates and washed in FACS buffer containing 3\% FBS, $1 \times$ PBS, and $2 \mathrm{mM}$ EDTA (vol/vol). After washing, cells were surface stained for 1 hour on ice to identify the hematopoietic cell population of interest (cells were stained in $50 \mu \mathrm{l} \mathrm{Ab}$ solution). Cells were washed once with $200 \mu \mathrm{l}$ FACS buffer and resuspended in $400 \mu \mathrm{l} \mathrm{FAC}$ $\mathrm{S}$ buffer for flow cytometric analysis.

The following anti-mouse Abs were used: phycoerythrin-conjugated (PE-conjugated) anti-B220 (BD Pharmingen; catalog 553090), anti-CD43 (BD Pharmingen; catalog 553271), anti-CD4 (eBioscience; catalog 12-0041-82), anti-CD8 (eBioscience; catalog 12-008182), anti-Ter119 (eBioscience; catalog 12-5921-81), anti-CD48 (BD Pharmingen; catalog 557485), anti-MAC1 (BD Pharmingen; cata$\log$ 557397), and anti-CD45.2 (BD Pharmingen; catalog 560695); PE-Cy7-linked anti-MAC1 (eBioscience; catalog 25-0112-82), antiIL-7R $\alpha$ (eBioscience; catalog 25-1271-82), anti-CD45.1 (eBioscience; catalog 25-0453-82), and streptavidin (eBioscience; catalog 25-4317-82); allophycocyanin-linked anti-B220 (eBioscience; catalog 17-0452-82), anti-Sca1 (eBioscience; catalog 17-5981-82), and anti-CD150 (eBioscience; catalog 17-1502-80); Pacific Blue-linked streptavidin (e450) (eBioscience; catalog 48-4317-82) and anti-CD93 (eBioscience; catalog 48-5892-80); FITC-conjugated anti-c-KIT (BD Pharmingen; catalog 553354); and biotin-linked anti-CD93 (eBioscience; catalog 13-5892-82). Flow cytometric analysis was performed on a CyAn, Cytomics FC 500, or Cell Lab Quanta SC flow cytometer (all from Beckman Coulter). B cell progenitors were defined on the basis of their expression of surface markers: pro-B cells: $\mathrm{B} 22 \mathrm{O}^{\mathrm{lo}}$, CD93 ${ }^{\text {hi }}, C D 43^{\text {hi }}$; pre-B cells: B220 ${ }^{\text {lo }}, \mathrm{CD} 93^{\text {hi }}, \mathrm{CD} 43^{\text {intermed/lo }}$; immature B cells: B220 $0^{\text {hi }}, \mathrm{CD} 93^{\text {neg }}, \mathrm{CD} 4^{\text {neg }}$. Cell sorting was performed on MoFlo XDP 70 and 100 cell sorters (Beckman Coulter).

Intracellular staining. Intracellular cytokine staining was performed as previously described (16). The following BD Biosciences PE-conjugated Abs were used: p-STAT3 (P-Y705, catalog 612569), p-STAT5 (P-Y694, catalog 612567), p-ERK1/2 (P-T202/P-Y204, catalog 612566), and control (rat IgG2b, catalog 556925). Anti-TNF- $\alpha$ $\mathrm{PE}$-conjugated $\mathrm{Ab}$ (BioLegend, catalog 506305) was used for the detection of TNF- $\alpha$. Stained cells were analyzed on the CyAn ADP Analyzer (Beckman Coulter).

\section{Statistics}

Unpaired $t$ tests, Cox proportional hazards tests, and 1-way ANOVA were used to analyze the data, with a $P$ value of less than 0.05 considered statistically significant. All error bars represent biological replicates (different mice), not technical replicates. Statistical analyses were performed with GraphPad Prism software, version 6.07 (GraphPad Software). All results are expressed as the mean \pm SEM.

\section{Study approval}

All animal experiments were approved by and performed in accordance with guidelines of the IACUC of the University of Colorado AMC.

\section{Author contributions}

$\mathrm{CJH}$ contributed to the study design, collected and interpreted data, created the manuscript figures, provided financial support for the study, and drafted and revised the manuscript. MCS assisted with the experiments depicted in Figure 1, D-G. J Kim, LA, and ACT provided bioinformatics assistance with the microarray data. VZ performed in vivo experiments and managed the mouse colonies. AED contributed to experiments shown in Figure 2, A and B. LJ performed the experiments presented in Supplemental Figure 1B. TA performed IL-1 $\beta$ ELISAs. ENM and ETC provided the TNF- $\alpha^{\triangle A R E}$ transgenic mice and proposed study designs. J Klawitter and NJS assisted CJH with mass spectrometry and the untargeted quantitative $1 \mathrm{H}$ NMR metabolomics experiments, respectively. CAD provided the AATtg and IL-37tg mice, proposed study designs, and reviewed the manuscript. JD conceived the study, provided financial support for the study, analyzed data, and assisted with the drafting of the manuscript. All authors read and approved the final manuscript.

\section{Acknowledgments}

These studies were supported by grants from the NIH (KO1 CA160798 and T32 AG000279, to C.J. Henry, R01 AI15614, to C.A. Dinarello, and R01 CA180175, to J. DeGregori); the UNCF/ Merck Science Initiative (2510259, to C.J. Henry); and the Interleukin Foundation (to C.A. Dinorello). The Metabolomics, Genomics and Flow Cytometry Shared Resources are supported by grant 5UL1-RR025780 from the National Center for Research Resources (NCRR) and the Colorado Clinical \& Translational Sciences Institute (CCTSI) and by Cancer Center Support grant P30-CA046934. We would also like to thank Kelly Higa, Andrii Rozhok, Raul Torres, and Craig T. Jordan of the University of Colorado AMC and Eric Pietras of the UCSF for their careful review of the manuscript.

Address correspondence to: Curtis J. Henry or James DeGregori, Mail Stop 8101, 12801 E. $1^{\text {th }}$ Ave., Room L18-9112, Aurora, Colorado 80045, USA. Phone: 303.724.3230; E-mail: James.DeGregori@ucdenver.edu (J. DeGregori), Curtis.Henry@ucdenver.edu (C.J. Henry).

Matias Casás-Selves's present address is: Ontario Institute for Cancer Research, Toronto, Ontario, Canada.

Ashley E. Daniel's present address is: Creighton University School of Medicine, Omaha, Nebraska, USA. 
1. DePinho RA. The age of cancer. Nature. 2000;408(6809):248-254.

2. Lopez-Otin C, Blasco MA, Partridge L, Serrano M, Kroemer G. The hallmarks of aging. Cell. 2013;153(6):1194-1217.

3. Chan GK, Duque G. Age-related bone loss: old bone, new facts. Gerontology. 2002;48(2):62-71.

4. Dykstra B, Olthof S, Schreuder J, Ritsema M, de Haan G. Clonal analysis reveals multiple functional defects of aged murine hematopoietic stem cells. JExp Med. 2011;208(13):2691-2703.

5. Rossi DJ, Bryder D, Weissman IL. Hematopoietic stem cell aging: mechanism and consequence. Exp Gerontol. 2007;42(5):385-390.

6. Pang WW, et al. Human bone marrow hematopoietic stem cells are increased in frequency and myeloid-biased with age. Proc Natl Acad Sci U S A. 2011;108(50):20012-20017.

7. Rossi DJ, et al. Cell intrinsic alterations underlie hematopoietic stem cell aging. Proc Natl Acad Sci U S A. 2005;102(26):9194-9199.

8. Geiger H, de Haan G, Florian MC. The ageing haematopoietic stem cell compartment. Nat Rev Immunol. 2013;13(5):376-389.

9. Snoeck HW. Aging of the hematopoietic system. Curr Opin Hematol. 2013;20(4):355-361.

10. Beerman I, et al. Functionally distinct hematopoietic stem cells modulate hematopoietic lineage potential during aging by a mechanism of clonal expansion. Proc Natl Acad Sci U S A. 2010;107(12):5465-5470.

11. Allman D, Miller JP. The aging of early B-cell precursors. Immunol Rev. 2005;205:18-29.

12. Linton PJ, Dorshkind K. Age-related changes in lymphocyte development and function. Nat Immunol. 2004;5(2):133-139.

13. Guerrettaz LM, Johnson SA, Cambier JC. Acquired hematopoietic stem cell defects determine B-cell repertoire changes associated with aging. Proc Natl Acad Sci U S A. 2008;105(33):11898-11902.

14. Riley RL. Impaired B lymphopoiesis in old age: a role for inflammatory B cells? Immunol Res. 2013;57(1-3):361-369.

15. Labrie JE 3rd, Sah AP, Allman DM, Cancro MP, Gerstein RM. Bone marrow microenvironmental changes underlie reduced RAG-mediated recombination and $\mathrm{B}$ cell generation in aged mice. J Exp Med. 2004;200(4):411-423.

16. Henry CJ, Marusyk A, Zaberezhnyy V, Adane $B$, DeGregori J. Declining lymphoid progenitor fitness promotes aging-associated leukemogenesis. Proc Natl Acad Sci U S A. 2010;107(50):21713-21718.

17. Pronk CJ, Veiby OP, Bryder D, Jacobsen SE. Tumor necrosis factor restricts hematopoietic stem cell activity in mice: involvement of two distinct receptors. J Exp Med.2011;208(8):1563-1570.

18. Challen GA, Boles NC, Chambers SM, Goodell MA. Distinct hematopoietic stem cell subtypes are differentially regulated by TGF- $\beta 1$. Cell Stem Cell. 2010;6(3):265-278.

19. Essers MA, et al. IFN $\alpha$ activates dormant haematopoietic stem cells in vivo. Nature. 2009;458(7240):904-908.

20. Baldridge MT, King KY, Boles NC, Weksberg DC, Goodell MA. Quiescent haematopoietic stem cells are activated by IFN- $\gamma$ in response to chronic infection. Nature. 2010;465(7299):793-797.

21. Pietras EM, et al. Re-entry into quiescence protects hematopoietic stem cells from the killing effect of chronic exposure to type I interferons. J Exp Med.2014;211(2):245-262.

22. Nagai $Y$, et al. Toll-like receptors on hematopoietic progenitor cells stimulate innate immune system replenishment. Immunity. 2006;24(6):801-812.

23. Ueda Y, Kondo M, Kelsoe G. Inflammation and the reciprocal production of granulocytes and lymphocytes in bone marrow. J Exp Med. 2005;201(11):1771-1780.

24. Ueda Y, Yang K, Foster SJ, Kondo M, Kelsoe G. Inflammation controls B lymphopoiesis by regulating chemokine CXCL12 expression. J Exp Med 2004;199(1):47-58.

25. Ratliff M, Alter S, Frasca D, Blomberg BB, Riley RL. In senescence, age-associated B cells secrete $\mathrm{TNF} \alpha$ and inhibit survival of B-cell precursors. Aging Cell. 2013;12(2):303-311.

26. Goto M. Inflammaging (inflammation + aging): a driving force for human aging based on an evolutionarily antagonistic pleiotropy theory? Biosci Trends. 2008;2(6):218-230.

27. Okin D, Medzhitov R. Evolution of inflammatory diseases. Curr Biol. 2012;22(17):R733-R740.

28. Michaud M, et al. Proinflammatory cytokines, aging, and age-related diseases. JAm Med Dir Assoc. 2013;14(12):877-882.

29. Giunta B, et al. Inflammaging as a prodrome to Alzheimer's disease. J Neuroinflammation. 2008;5:51.

30. Lencel P, Magne D. Inflammaging: the driving force in osteoporosis? Med Hypotheses. 2011;76(3):317-321.

31. Franceschi C, et al. Inflamm-aging. An evolution ary perspective on immunosenescence. Ann N Y Acad Sci. 2000;908:244-254.

32. Collado M, Blasco MA, Serrano M. Cellular senescence in cancer and aging. Cell. 2007;130(2):223-233.

33. Hoeijmakers JH. DNA damage, aging, and cancer. N Engl J Med. 2009;361(15):1475-1485.

34. Vogelstein B, Papadopoulos N, Velculescu VE, Zhou S, Diaz LA Jr, Kinzler KW. Cancer genome landscapes. Science. 2013;339(6127):1546-1558.

35. Rozhok AI, Salstrom JL, DeGregori J. Stochastic modeling indicates that aging and somatic evolution in the hematopoetic system are driven by non-cell-autonomous processes. Aging. 2014;6(12):1033-1048.

36. Vijg J, Busuttil RA, Bahar R, Dolle ME. Aging and genome maintenance. Ann N Y Acad Sci. 2005;1055:35-47.

37. Miller JP, Allman D. The decline in B lymphopoiesis in aged mice reflects loss of very early B-lineage precursors. JImmunol. 2003;171(5):2326-2330.

38. Dias S, Silva H Jr, Cumano A, Vieira P. Interleukin-7 is necessary to maintain the $B$ cell potential in common lymphoid progenitors. J Exp Med. 2005;201(6):971-979.

39. Flach J, et al. Replication stress is a potent driver of functional decline in ageing haematopoietic stem cells. Nature. 2014;512(7513):198-202.

40. Miller JP, Allman D. Linking age-related defects in B lymphopoiesis to the aging of hematopoietic stem cells. Semin Immunol. 2005;17(5):321-329.

41. Ren R. Mechanisms of BCR-ABL in the pathogen- esis of chronic myelogenous leukaemia. Nat Rev Cancer. 2005;5(3):172-183.

42. Mirantes C, Passegué E, Pietras EM. Pro-inflammatory cytokines: emerging players regulating HSC function in normal and diseased hematopoiesis. Exp Cell Res. 2014;329(2):248-254.

43. Casalino-Matsuda SM, Monzon ME, Day AJ, Forteza RM. Hyaluronan fragments/CD44 mediate oxidative stress-induced MUC5B up-regulation in airway epithelium. Am J Respir Cell Mol Biol. 2009;40(3):277-285.

44. Kontoyiannis D, Pasparakis M, Pizarro TT, Cominelli F, Kollias G. Impaired on/off regulation of TNF biosynthesis in mice lacking TNF AU-rich elements: implications for joint and gut-associated immunopathologies. Immunity. 1999;10(3):387-398.

45. McNamee EN, et al. Chemokine receptor CCR7 regulates the intestinal $\mathrm{TH1} / \mathrm{TH} 17 /$ Treg balance during Crohn's-like murine ileitis. J Leukoc Biol. 2015;97(6):1011-1022.

46. Lewis EC, et al. $\alpha 1$-Antitrypsin monotherapy induces immune tolerance during islet allograft transplantation in mice. Proc Natl Acad Sci U S A. 2008;105(42):16236-16241.

47. Pott GB, Chan ED, Dinarello CA, Shapiro L. $\alpha 1$-Antitrypsin is an endogenous inhibitor of proinflammatory cytokine production in whole blood. J Leukoc Biol. 2009;85(5):886-895.

48. Lewis EC. Expanding the clinical indications for $\alpha(1)$-antitrypsin therapy. Mol Med. 2012;18:957-970.

49. Dinarello CA, Kim S, Bufler P. IL-37: the IL-1 family member you never expected. Eur J Immunol. In press.

50. Garlanda C, Dinarello CA, Mantovani A. The interleukin-1 family: back to the future. Immunity. 2013;39(6):1003-1018.

51. Nold MF, Nold-Petry CA, Zepp JA, Palmer BE, Bufler P, Dinarello CA. IL-37 is a fundamental inhibitor of innate immunity. Nat Immunol. 2010;11(11):1014-1022.

52. Oh J, Lee YD, Wagers AJ. Stem cell aging: mechanisms, regulators and therapeutic opportunities. Nat Med. 2014;20(8):870-880.

53. Conboy MJ, Conboy IM, Rando TA. Heterochronic parabiosis: historical perspective and methodological considerations for studies of aging and longevity. Aging Cell. 2013;12(3):525-530.

54. Cheleuitte D, Mizuno S, Glowacki J. In vitro secretion of cytokines by human bone marrow: effects of age and estrogen status. JClin Endocrinol Metab. 1998;83(6):2043-2051.

55. Welner RS, et al. Treatment of chronic myelogenous leukemia by blocking cytokine alterations found in normal stem and progenitor cells. Cancer Cell. 2015;27(5):671-681.

56. Reynaud D, et al. IL-6 controls leukemic multipotent progenitor cell fate and contributes to chronic myelogenous leukemia development. Cancer Cell. 2011;20(5):661-673.

57. Kleppe M, et al. JAK-STAT pathway activation in malignant and nonmalignant cells contributes to MPN pathogenesis and therapeutic response. Cancer Discov. 2015;5(3):316-331.

58. Min H, Montecino-Rodriguez E, Dorshkind K. Effects of aging on early B- and T-cell development. Immunol Rev. 2005;205:7-17. 
59. Geiger H, Zheng Y. Cdc42 and aging of hematopoietic stem cells. Curr Opin Hematol. 2013;20(4):295-300.

60. Chambers SM, Shaw CA, Gatza C, Fisk CJ, Donehower LA, Goodell MA. Aging hematopoietic stem cells decline in function and exhibit epigenetic dysregulation. PLoS Biol. 2007;5(8):e201.

61. Cullen SM, Mayle A, Rossi L, Goodell MA. Hematopoietic stem cell development: an epigenetic journey. Curr Top Dev Biol. 2014;107:39-75.

62. Walter D, et al. Exit from dormancy provokes DNA-damage-induced attrition in haematopoietic stem cells. Nature. 2015;520(7548):549-552.

63. Frith $\mathrm{CH}$, Ward JM, Chandra M. The morphology, immunohistochemistry, and incidence of hematopoietic neoplasms in mice and rats. Toxicol Pathol. 1993;21(2):206-218.

64. Henry CJ, Marusyk A, DeGregori J. Agingassociated changes in hematopoiesis and leukemogenesis: what's the connection? Aging. 2011;3(6):643-656.

65. Greaves M. Infection, immune responses and the aetiology of childhood leukaemia. Nat Rev Cancer. 2006;6(3):193-203.

66. Coussens LM, Werb Z. Inflammation and cancer. Nature. 2002;420(6917):860-867.

67. Lin WW, Karin M. A cytokine-mediated link between innate immunity, inflammation, and cancer. JClin Invest. 2007;117(5):1175-1183.

68. Jaiswal S, et al. Age-related clonal hematopoiesis associated with adverse outcomes. $N$ Engl JMed. 2014;371(26):2488-2498.
69. Genovese G, et al. Clonal hematopoiesis and blood-cancer risk inferred from blood DNA sequence. $N$ EnglJMed. 2014;371(26):2477-2487.

70. Xie M, et al. Age-related mutations associated with clonal hematopoietic expansion and malignancies. Nat Med. 2014;20(12):1472-1478.

71. Jacobs KB, et al. Detectable clonal mosaicism and its relationship to aging and cancer. Nat Genet. 2012;44(6):651-658.

72. Laurie CC, et al. Detectable clonal mosaicism from birth to old age and its relationship to cancer. Nat Genet. 2012;44(6):642-650.

73. Hasselbalch HC. Perspectives on chronic inflammation in essential thrombocythemia, polycythemia vera, and myelofibrosis: is chronic inflammation a trigger and driver of clonal evolution and development of accelerated atherosclerosis and second cancer? Blood. 2012;119(14):3219-3225.

74. Kralovics R, et al. A gain-of-function mutation of JAK2 in myeloproliferative disorders. $N$ Engl J Med. 2005;352(17):1779-1790.

75. Vas V, Wandhoff C, Dorr K, Niebel A, Geiger H. Contribution of an aged microenvironment to aging-associated myeloproliferative disease. PLoS One. 2012;7(2):e31523.

76. Vas V, Senger K, Dorr K, Niebel A, Geiger $\mathrm{H}$. Aging of the microenvironment influences clonality in hematopoiesis. PLoS One. 2012;7(8):e42080.

77. Kristinsson SY, Bjorkholm M, Hultcrantz M, Derolf AR, Landgren O, Goldin LR. Chronic immune stimulation might act as a trigger for the development of acute myeloid leukemia or myelodysplastic syndromes. J Clin Oncol. 2011;29(21):2897-2903.

78. Cuzick J, et al. Estimates of benefits and harms of prophylactic use of aspirin in the general population. Ann Oncol. 2015;26(1):47-57.

79. Streicher SA, Yu H, Lu L, Kidd MS, Risch HA. Case-control study of aspirin use and risk of pancreatic cancer. Cancer Epidemiol Biomarkers Prev. 2014;23(7):1254-1263.

80. Neill AS, Nagle CM, Protani MM, Obermair A, Spurdle AB, Webb PM. Aspirin, nonsteroidal anti-inflammatory drugs, paracetamol and risk of endometrial cancer: a case-control study, systematic review and meta-analysis. Int J Cancer. 2013;132(5):1146-1155.

81. Navarrete-Reyes AP, Montana-Alvarez M. [Inflammaging. Aging inflammatory origin]. Rev Invest Clin. 2009;61(4):327-336.

82. Franceschi C, et al. Genes involved in immune response/inflammation, IGF1/insulin pathway and response to oxidative stress play a major role in the genetics of human longevity: the lesson of centenarians. Mech Ageing Dev. 2005;126(2):351-361.

83. DeGregori J. Challenging the axiom: does the occurrence of oncogenic mutations truly limit cancer development with age? Oncogene. 2013;32(15):1869-1875.

84. Bilousova G, Marusyk A, Porter CC, Cardiff RD, DeGregori J. Impaired DNA replication within progenitor cell pools promotes leukemogenesis. PLoS Biol. 2005;3(12):e401. 